\title{
LOCAL CIP STABILIZATION FOR COMPOSITE FINITE ELEMENTS*
}

\author{
ERIK BURMAN ${ }^{\dagger}$ AND FRIEDHELM SCHIEWECK ${ }^{\ddagger}$
}

This paper is dedicated to the "doctor father" and longtime companion Prof. Dr. Herbert

Goering of the second author (F. Schieweck) on the occasion of his 90th birthday in

gratitude and respect for his openness in passing his wisdom of life and his philosophy of

knowledge acquisition to his students

\begin{abstract}
We propose a continuous interior penalty (CIP) method for the pure transport problem and for the viscosity dependent "Stokes-Brinkman" problem where the gradient jump penalty is localized to faces in the interior of subdomains. Special focus is given to the case where the subdomains are so-called composite finite elements, e.g., quadrilateral, hexahedral or prismatic elements which are composed by simplices such that the arising global simplicial mesh is regular. The advantage of this local CIP is that it allows for static condensation in contrast to the classical CIP method. If the degrees of freedom in the interior of the composite finite elements are eliminated using static condensation then the resulting couplings of the skeleton degrees of freedom are comparable to those for classical conforming finite element methods which leads to a substantially smaller matrix stencil than for the standard global CIP method. Optimal stability and error estimates are proved and numerical tests are presented. For the Stokes-Brinkman model, our error bound does not increase if the viscosity parameter tends to zero which is mainly achieved by adding a penalty term for the divergence of the velocity in the discretization. Moreover, the reduction effect of the static condensation is much stronger for this model since, beside the elimination of all velocity degrees of freedom in the interior of each composite cell, all pressure degrees of freedom except for the cellwise constants can be eliminated.
\end{abstract}

Key words. stabilization, transport equation, Stokes-Brinkman equation, finite element method, composite elements

AMS subject classifications. 65N30, 35J25, 76M10

DOI. $10.1137 / 15 \mathrm{M} 1039390$

1. Introduction. The last fifteen years have seen a rapid development of symmetric stabilization methods for convection-diffusion equations using globally continuous finite element spaces, or for Stokes' equations discretized using globally continuous equal order interpolation for velocities and pressures. Early works in this direction include the subgrid viscosity method suggested by Guermond [13], the orthogonal subscales introduced by Codina [11], and the minimal stabilization approaches for mixed finite element methods discussed by Brezzi and Fortin [4]. In the subgrid viscosity method an artificial viscosity term is added that is made to act on the finest scales of the problem only by introducing two scales in the finite element space, either by adding degrees of freedom (dofs) in the computational mesh or by adding higher order polynomials in the finite element space. In the orthogonal subscale stabilization on the other hand a global projection of the gradient is subtracted from the gradient in the artificial viscosity term, making the diffusion act only on the finest scales.

The field then expanded rapidly with the introduction of the local projection stabilization by Becker and Braack [1,2] and the continuous interior penalty (CIP)

*Received by the editors September 11, 2015; accepted for publication (in revised form) March 28, 2016; published electronically June 29, 2016.

http://www.siam.org/journals/sinum/54-3/M103939.html

$\dagger$ Department of Mathematics, University College London, London, UK-WC1E 6BT, United Kingdom (e.burman@ucl.ac.uk).

${ }^{\ddagger}$ Institut für Analysis und Numerik, Otto-von-Guericke Universität Magdeburg, D-39016 Magdeburg, Germany (schiewec@ovgu.de). 
method suggested by Burman and Hansbo $[8,9]$. In the former of these methods a local projection of the gradient on element patches is subtracted from the gradient in the viscosity operator, leading to a more local type of scheme than the orthogonal subscale method. In the CIP method, on the other hand, an $L^{2}$-penalty term on the jump of the gradient over all element faces is added to the formulation. Another type of symmetric stabilization based on interpolation of the gradient was recently proposed and analyzed in [10]. For further details on stabilized methods for convection-diffusion equations we refer to the monograph [16] and references therein.

All the above methods can typically be classified according to the locality of the stabilization, some methods act on some a priori defined subgrid/subspace, such as the subgrid viscosity or the local projection stabilization, whereas others such as the orthogonal subscales or the CIP method use a stabilization that is not localized but acts globally. Both approaches can be argued to have their advantages and there are some clear similarities between some of the methods. For instance the local projection stabilization can both be seen as a local variant of the orthogonal subscale method and in special cases it coincides with the subgrid viscosity method, as discussed in [14]. The situation is less clear for the CIP method. Although the method has been used on macropatches in some works, [17] (Stokes) and [15] (convection diffusion), stability has been a consequence of the close relation to other methods, namely, the inf-sup stability of an underlying stable velocity-pressure pair in [17] and the equivalence with subgrid viscosity in [15].

In this paper our aim is to discuss local CIP stabilization in a more general setting both for stabilization of advection and the Stokes-Brinkman equations. Using finite element spaces of arbitrary polynomial order we prove that the CIP stabilization may be restricted to subdomains without loss of stability. The only requirement on the subdomains is that every triangle of a subdomain has at least one vertex in the interior of this subdomain.

The results herein are of interest in several contexts. For instance it shows that strongly imposed boundary conditions may be used together with CIP stabilization for all polynomial orders. Another consequence of the analysis is that when domain decomposition is used for the solution of the linear system no stabilization is needed across subdomain boundaries, allowing for standard $C^{0}$ domain decomposition algorithms to be applied. It also allows for the use of pressure spaces that are discontinuous over subdomain boundaries leading to enhanced mass conservation on the subdomain level.

In this work we present the method in the context of composite finite elements. That is a finite element method, where the starting mesh can consist of arbitrary star-shaped polyhedral cells. From this starting mesh a final global simplicial mesh is created automatically by applying simple decomposition rules locally on each cell such that the resulting global simplicial mesh is regular (without hanging nodes). The advantage is that, for more complicated polyhedral cells, a complicated reference transformation to the reference element can be avoided and that the classical polynomial $\mathbb{P}_{k}$-functions with total degree not greater than $k$ can be used, which implies, for instance, that each differentiation decreases the polynomial degree by one. The most important advantage of the local CIP method compared to the classical one concerning the computational costs is the fact that here the dofs in the interior of a composite cell are only coupled with the dofs at their cell boundary and decoupled from the dofs in the interior of other composite cells. Thus, all interior nodes may be eliminated using static condensation leading to a stabilized method with dofs only on the macro-mesh-skeleton and with only next-neighbor couplings between the skele- 


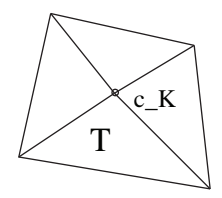

FIG. 1. Quadrilateral element $K \in \mathcal{M}_{h}$ is composed by simplicial elements $T \in \mathcal{T}_{K}$.

ton dofs as in the standard Galerkin method. In contrast, the static condensation is impossible for the classical CIP method. Therefore, the local CIP method avoids the negative effect of the extended stencil in the classical CIP method and reduces essentially the computational costs regarding storage and CPU time.

The rest of the paper can be outlined as follows. First we will discuss the composite elements that we use in the analysis and the associated finite element spaces. Then we consider the case of a first order hyperbolic transport problem and prove discrete stability in the form of an inf-sup condition and error estimates for the local CIP method defined on the composite elements. We finally consider the Stokes-Brinkman equations. Also in this case we prove a discrete inf-sup condition and optimal error estimates, expliciting the dependence on the viscosity showing that the method can also be applied in the case of high Reynolds number. The paper is concluded with some numerical experiments.

General notation. For a measurable subset $G \subset \mathbb{R}^{d}$, let $L^{p}(G), 1 \leq p \leq \infty$, $W^{s, p}(G), s \geq 1$, denote the standard Sobolev spaces and $H^{s}(G):=W^{s, 2}(G)$ the Hilbert space with the seminorm $|\cdot|_{s, G}$. We denote by $\|\cdot\|_{G}$ and $(\cdot, \cdot)_{G}$ the norm and inner product in $L^{2}(G)$, respectively. If $G$ is a $(d-1)$-dimensional face of an element or a union of faces, the space $L^{2}(G)$ has to be understood in the sense of traces and we denote by $\langle\cdot, \cdot\rangle_{G}$ the inner product and by $\|\cdot\|_{G}$ the associated norm. In a context-sensitive way, we use the above notation also for vector-valued functions in $L^{2}(G)^{d}$ or $H^{s}(G)^{d}$, respectively. The notation $C$ indicates a general constant which can have different values at different places but which is independent of the local and global mesh size.

2. Composite elements. To define "composite elements" we start with a socalled "macromesh" $\mathcal{M}_{h}$ of the domain $\Omega \subset \mathbb{R}^{d}$ consisting of simple macroelements $K \in \mathcal{M}_{h}$, where $K$ can be any star-shaped polyhedral cell, for instance, a quadrilateral or triangle in 2 dimensions or a hexahedron, prism, or tetrahedron in 3 dimensions. We call the elements $K \in \mathcal{M}_{h}$ "composite elements" since they are composed by a relatively small number of simplicial subelements $T \in \mathcal{T}_{K}$ by taking one center point $c_{K} \in K$ and connecting $c_{K}$ by means of edges with the boundary of $K$ (see Figure 1). In the three-dimensional (3D) case, we first have to decompose the two-dimensional (2D) faces into triangles by drawing diagonals such that the number of vertices is not increasing. Thus, the domain $\Omega$ is decomposed as

$$
\bar{\Omega}=\bigcup_{K \in \mathcal{M}_{h}} \bar{K}, \quad \text { where } \quad \bar{K}=\bigcup_{T \in \mathcal{T}_{K}} \bar{T},
$$

as well as $\bar{\Omega}=\bigcup_{T \in \mathcal{T}_{h}} \bar{T}$, where $\mathcal{T}_{h}:=\bigcup_{K \in \mathcal{M}_{h}} \mathcal{T}_{K}$ denotes the final simplicial mesh. We assume that the simplicial mesh $\mathcal{T}_{h}$ is admissible (no hanging vertices) and shape regular, i.e., it holds that $\rho_{T} \geq C h_{T}$ for all $T \in \mathcal{T}_{h}$, where $h_{T}$ denotes the diameter of $T$ and $\rho_{T}$ the radius of the largest ball that can be inscribed into $T$. For a composite element $K \in \mathcal{M}_{h}$, we denote by $h_{K}$ the maximum of the diameters $h_{T}$ of all 


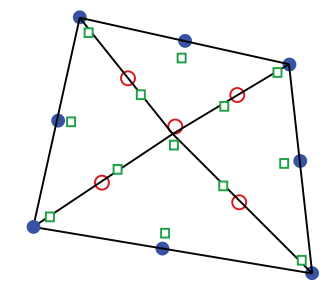

$4 \mathbb{P}_{2}^{\mathrm{c}} \times 4 \mathbb{P}_{2}^{\mathrm{c}}$ composite dofs

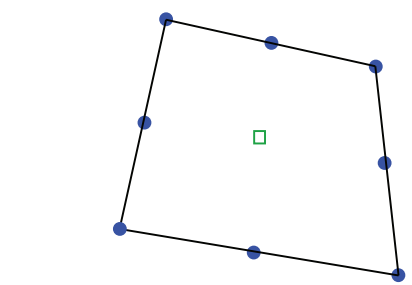

after static condensation $\sim \mathbb{Q}_{2}$-dofs

FIG. 2. Example of degrees of freedom on a piecewise quadratic composite element, before and after static condensation. We consider both continuous velocity approximation and pressure approximation that is discontinuous across macro element boundaries: velocity dofs: $\circ$ (interior) $+\bullet$ (skeleton) pressure dofs: $\square$.

subelements $T \in \mathcal{T}_{K}$. From the shape regularity of $\mathcal{T}_{h}$ and the fact that the number of subelements in $\mathcal{T}_{K}$ is uniformly bounded we get the mesh property

$$
C h_{K} \leq h_{T} \leq h_{K} \quad \forall T \in \mathcal{T}_{K}, \quad K \in \mathcal{M}_{h},
$$

where the constant $C$ is independent of $T$ and $K$. For a given composite element $K \in \mathcal{M}_{h}$, we will denote in the following by $\mathcal{F}_{K}$ the set of those $(d-1)$-dimensional faces $F$ of the subelements $T \in \mathcal{T}_{K}$, where $F$ is located in the interior of $K$.

2.1. Advantages of composite elements. In this subsection, we will discuss the advantages of using composite elements. The first advantage is that the framework of composite elements allows us to construct meshes that can contain arbitrary starshaped polyhedral mesh cells which improves the flexibility for mesh generators.

Since the final mesh $\mathcal{T}_{h}$ is always a simplicial mesh, the question arises why we do not work only with the simplicial mesh? The answer is that a simplicial mesh needs, in particular in the 3D case, many more elements than, for instance, a hexahedral mesh (the factor is at least 5). To administrate a quadrilateral or hexahedral macromesh $\mathcal{M}_{h}$ is often cheaper than a simplicial final mesh $\mathcal{T}_{h}$, in particular, with respect to adaptive refinements in 3 dimensions.

When the local stiffness matrix $A^{K}$ for a composite element $K \in \mathcal{M}_{h}$ has to be computed, the geometrical data of the polyhedral cell $K$ is loaded and the geometrical data for the simplices $T \in \mathcal{T}_{K}$ are easily computed. Then, a loop over the small number of simplices $T \in \mathcal{T}_{K}$ is executed with the standard procedure for generating the local element matrix $A_{T}^{K}$ for a simplicial element $T$. The entries of $A_{T}^{K}$ are added into the stiffness matrix $A^{K}$. Also the jump terms from the local CIP method over the interior simplex faces $F \in \mathcal{F}_{K}$ can be generated during the local simplex loop more efficiently than for the classical CIP method. Once the local stiffness matrix $A^{K}$ and the related local right-hand side vector $F^{K}$ are computed, the dofs in the interior of $K$ can be eliminated by computing the local Schur complement. Only the entries of the Schur complement are added into the global sparse stiffness matrix. This essentially improves the sparsity pattern of the stiffness matrix compared to the classical CIP method. Composite elements are well suited for implementing static condensation. Note that the higher computational efficiency of using composite elements instead of the final simplicial mesh $\mathcal{T}_{h}$ is only achieved if the static condensation is exploited (see Figure 2).

Finally, the framework of composite elements offers the following advantage for adaptively refined meshes. One can use an adaptive finite element approach that is 

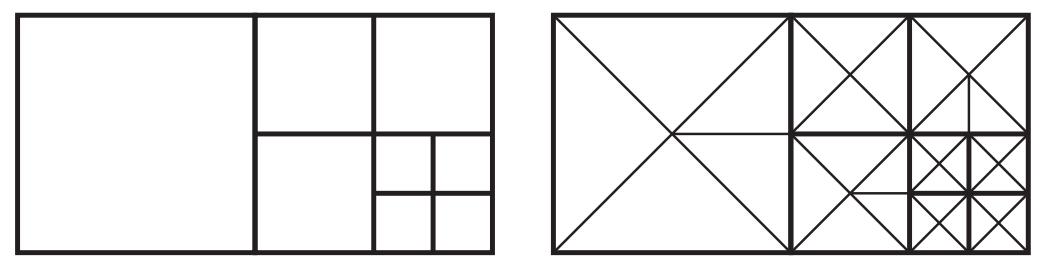

FIG. 3. Adaptively refined macromesh $\mathcal{M}_{h}$ with hanging nodes (left) and adaptively refined composite mesh $\mathcal{T}_{h}$ without hanging nodes (right).

designed for the use of hanging nodes in the macromesh $\mathcal{M}_{h}$. From such a mesh $\mathcal{M}_{h}$, there can be easily generated a final simplicial mesh $\mathcal{T}_{h}$ that does not have hanging nodes. We will illustrate the idea for a $2 \mathrm{D}$ situation.

Let $\mathcal{M}_{0}$ be an admissible coarse macromesh, i.e., without any hanging nodes. Then, define recursively the next mesh $\mathcal{M}_{\ell+1}$ from the previous one $\mathcal{M}_{\ell}$ by adaptive refinement in such a way that the difference of the refinement level of adjacent composite elements is at most one (see Figure 3). Consequently, each macromesh $\mathcal{M}_{h}$ in the sequence of adaptive multilevel grids contains hanging nodes in general. To form the subelement decomposition of a macrocell $K \in \mathcal{M}_{h}=\mathcal{M}_{\ell+1}$ we connect the center point $c_{K} \in K$ by means of edges with the vertices at the boundary of $K$ which can also be "hanging vertices" from an adjacent macrocell $K^{\prime} \in \mathcal{M}_{h}$ (see Figure 3 ). Then the final mesh $\mathcal{T}_{h}$ no longer contains a hanging node.

2.2. Local spaces and averaging operator. For a nonnegative integer $m$ and a given composite element $K \in \mathcal{M}_{h}$, we introduce the following discontinuous and continuous composite polynomial spaces:

$$
\begin{aligned}
& D_{m}(K):=\left\{w \in L^{2}(K):\left.w\right|_{T} \in \mathbb{P}_{m}(T) \forall T \in \mathcal{T}_{K}\right\}, \\
& U_{m}(K):=D_{m}(K) \cap C^{0}(\bar{K}),
\end{aligned}
$$

and define a local averaging operator $I_{\mathrm{av}}^{K, m}: D_{m}(K) \rightarrow U_{m}(K)$ as follows. For a given function $w \in D_{m}(K)$, we define $I_{\mathrm{av}}^{K, m} w \in U_{m}(K)$ by its values at a corresponding set of nodal points $a_{j} \in \bar{K}, j \in J(K)$. Let $\mathcal{T}_{K}^{j}:=\left\{T \in \mathcal{T}_{K}: a_{j} \in \bar{T}\right\}$ denote the subset of all simplices $T \subset K$ that share the nodal point $a_{j}$. Then we define the nodal value of $I_{\mathrm{av}}^{K, m} w$ at $a_{j}$ as the mean value

$$
I_{\mathrm{av}}^{K, m} w\left(a_{j}\right):=\left.\left(\operatorname{card}\left(\mathcal{T}_{K}^{j}\right)\right)^{-1} \sum_{T \in \mathcal{T}_{K}^{j}} w\right|_{T}\left(a_{j}\right) .
$$

From [7, Lemma 5.3] we obtain for $m \geq 0$ and all $K \in \mathcal{M}_{h}$ the estimate

$$
\left\|w-I_{\mathrm{av}}^{K, m} w\right\|_{T} \leq C(m) h_{K}^{1 / 2} \sum_{F \in \mathcal{F}_{K}}\left\|\llbracket w \rrbracket_{F}\right\|_{F} \quad \forall w \in D_{m}(K), \quad T \in \mathcal{T}_{K} .
$$

Where $\llbracket w \rrbracket_{F}$ denotes the jump of the quantity $w$ over the face $F$. Using the triangle inequality, (2.3), (3.12), and an inverse inequality, we obtain for $m \geq 0$ the $L^{2}$-stability estimate

$$
\left\|I_{\mathrm{av}}^{K, m} w\right\|_{K} \leq C(m)\|w\|_{K} \quad \forall w \in D_{m}(K), \quad K \in \mathcal{M}_{h} .
$$

2.3. Composite $\mathbb{P}_{1}$-bubble functions. An essential key ingredient in the proof of the inf-sup stability of the local CIP discretization is the use of composite $\mathbb{P}_{1}$-bubble 
functions $\xi_{K}$ as multipliers for piecewise discontinuos discrete streamline derivatives or pressure gradients. Since these $\mathbb{P}_{1}$-bubble functions vanish at the boundary of each macroelement $K \in \mathcal{M}_{h}$, the constructed product function does not have jumps across the macroelement boundaries.

To this end, we assign to each composite element $K \in \mathcal{M}_{h}$ the continuous $\mathbb{P}_{1^{-}}$ bubble function $\xi_{K} \in U_{1}(K)$ which is defined by the conditions $\xi_{K}\left(c_{K}\right)=1$ and $\xi_{K}(x)=0$ for all $x \in \partial K$. From the property $0 \leq \xi_{K}(x) \leq 1$ for all $x \in K$ we get the following norm equivalence.

Lemma 2.1. For the above defined function $\xi_{K} \in U_{1}(K)$, a given exponent $p>0$, and a polynomial degree $m \geq 0$, there exists a constant $C_{1}(m, p)>0$ such that

$$
C_{1}(m, p)\left\|w_{h}\right\|_{K}^{2} \leq\left\|\xi_{K}^{p} w_{h}\right\|_{K}^{2} \leq\left\|w_{h}\right\|_{K}^{2} \quad \forall w_{h} \in D_{m}(K), K \in \mathcal{M}_{h} .
$$

Proof. The upper estimate simply follows from $\xi_{K}^{2 p} \leq 1$. To prove the lower estimate we introduce for each subelement $T \in \mathcal{T}_{K}$ the affine reference mapping $R_{T}: \hat{T} \rightarrow T$, where $\hat{T}$ denotes the reference simplex. Let $\hat{\xi}:=\xi_{K} \circ R_{T} \in \mathbb{P}_{1}(\hat{T})$. From $\hat{\xi}>0$ on $\operatorname{int}(\hat{T})$ we deduce that $\hat{w} \mapsto\left(\int_{\hat{T}}\left(\hat{\xi}^{p} \hat{w}\right)^{2} d \hat{x}\right)^{1 / 2}$ is a norm for all $\hat{w} \in$ $\mathbb{P}_{m}(\hat{T})$. Due to norm equivalence there exists a constant $C_{1}(m, p)>0$ such that $\int_{\hat{T}}\left(\hat{\xi}^{p} \hat{w}\right)^{2} d \hat{x} \geq C_{1}(m, p) \int_{\hat{T}} \hat{w}^{2} d \hat{x}$ for all $\hat{w} \in \mathbb{P}_{m}(\hat{T})$. Using this estimate and the fact that the Jacobian $J_{T}:=\operatorname{det}\left(D R_{T}\right)$ of the affine transformation $R_{T}$ is constant on $\hat{T}$ we obtain for each $w_{h} \in D_{m}(K)$ by means of transformation of integrals

$$
\left\|\xi_{K}^{p} w_{h}\right\|_{K}^{2} \geq \sum_{T \in \mathcal{T}_{K}} C_{1}(m, p) \int_{\hat{T}}\left(w_{h} \circ R_{T}\right)^{2}\left|J_{T}\right| d \hat{x}=C_{1}(m, p)\left\|w_{h}\right\|_{K}^{2} \cdot
$$

\section{Local CIP discretization for the transport problem.}

3.1. The transport problem. Let $\Omega$ be an open bounded and connected domain in $\mathbb{R}^{d}, d \leq 3$, with a polygonal boundary $\Gamma$ and outer normal $n$. As the data of our problem let $\beta \in W^{1, \infty}(\Omega)^{d}$ be a vector field, $\sigma \in L^{\infty}(\Omega)$, and $f \in L^{2}(\Omega)$. Then we consider the following model problem: Find $u: \Omega \rightarrow \mathbb{R}$ such that

$$
\begin{aligned}
\beta \cdot \nabla u+\sigma u & =f & & \text { in } \Omega, \\
u & =0 & & \text { on } \Gamma^{-},
\end{aligned}
$$

where $\Gamma^{-}:=\{x \in \partial \Omega: \beta \cdot n<0\}$. For our analysis, we assume the usual condition that the data satisfy

$$
\sigma(x)-\frac{1}{2} \operatorname{div} \beta(x) \geq \mu_{0}>0 \quad \forall x \in \Omega .
$$

Introducing the solution space $V:=\left\{v \in L^{2}(\Omega): \beta \cdot \nabla v \in L^{2}(\Omega)\right\}$ and the bilinear form $a$ on $V \times V$ defined by

$$
a(u, v):=(\beta \cdot \nabla u, v)_{\Omega}+(\sigma u, v)_{\Omega}+\langle|\beta \cdot n| u, v\rangle_{\Gamma^{-}},
$$

the weak formulation of the problem reads as follows: Find $u \in V$ such that

$$
a(u, v)=(f, v) \quad \forall v \in V .
$$

The essential condition that guarantees existence and uniqueness of a soution of problem (3.3) is (3.2); for details we refer to [12, Example 5.2.3]. 
3.2. Local CIP discretization. We choose a conforming finite element space $V_{h}$ defined as

$$
V_{h}:=\left\{v \in H^{1}(\Omega):\left.v\right|_{T} \in \mathbb{P}_{r}(T) \quad \forall T \in \mathcal{T}_{K}, K \in \mathcal{M}_{h}\right\},
$$

where $r \geq 1$ denotes the polynomial order of $V_{h}$. Let $W_{h}$ denote the space $W_{h}:=$ $H^{2}(\Omega)+V_{h}$. Then, in the local CIP stabilization method, the following modified bilinear form $a_{h}: W_{h} \times W_{h} \rightarrow \mathbb{R}$ is used,

$$
a_{h}(u, v):=a(u, v)+\sum_{K \in \mathcal{M}_{h}} j_{K}(u, v),
$$

where, for each macrocell $K$, the local jump term

$$
j_{K}(u, v):=\gamma_{0} h_{K}^{2} \sum_{F \in \mathcal{F}_{K}}\left|\beta_{K} \cdot n_{F}\right|\left\langle\llbracket \nabla u \rrbracket_{F}, \llbracket \nabla v \rrbracket_{F}\right\rangle_{F}
$$

is added, which penalizes the situation when the gradient of the discrete solution is not continuous across the faces in the interior of $K$. Here, $\beta_{K}:=\beta\left(c_{K}\right)$ (where $c_{K}$ is the center point of $K$ that defines the simplicial decomposition of $K$ ) and $\gamma_{0} \geq C \beta_{\infty}$ (with $\beta_{\infty}:=\|\beta\|_{L^{\infty}(\Omega)^{d}}$ ) denotes a user-chosen parameter independent of $h$.

Now, the discrete problem reads as follows: Find $u_{h} \in V_{h}$ such that

$$
a_{h}\left(u_{h}, v_{h}\right)=\left(f, v_{h}\right) \quad \forall v_{h} \in V_{h} .
$$

\subsection{Error analysis.}

3.3.1. Discrete norms. For a function $v \in V_{h}+H^{2}(\Omega)$ and an arbitrary composite element $K \in \mathcal{M}_{h}$, we introduce the local norms

$$
\|v\|_{K}^{2}:=\mu_{0}\|v\|_{K}^{2}+\frac{1}{2}\left\||\beta \cdot n|^{\frac{1}{2}} v\right\|_{\partial K \cap \Gamma}^{2}+j_{K}(v, v)
$$

with $\mu_{0}>0$ from (3.2) and

$$
\|v\|_{S, K}^{2}:=\|v\|_{K}^{2}+h_{K}\|\beta \cdot \nabla v\|_{K}^{2},
$$

where the symbol $S$ indicates "strong" or "streamline derivative" norm. To these local norms we assign the corresponding global norms

$$
\|v\|^{2}:=\sum_{K \in \mathcal{M}_{h}}\|v\|_{K}^{2} \quad \text { and } \quad\|v\|_{S}^{2}:=\sum_{K \in \mathcal{M}_{h}}\|v\|_{S, K}^{2} .
$$

In the following, we will derive some estimates for the local norms $\|\cdot\|_{K}$ and $\|\cdot\|_{S, K}$. Let $F \in \mathcal{F}_{K}$ be an interior face of the macroelement $K$ and $T \in \mathcal{T}_{K}$ a subelement of $K$ such that $F \subset \partial T$. Then, by means of standard arguments (transformation of $T$ to the reference element $\hat{T}$, trace theorem on $\hat{T}$, and transformation back to $T$ ) and the mesh property (2.1) we get for $m \geq 0$ the estimate

$$
\left\|\left.v\right|_{T}\right\|_{F}^{2} \leq C\left\{h_{K}^{-1}\|v\|_{T}^{2}+h_{K}|v|_{1, T}^{2}\right\} \quad \forall v \in H^{1}(T) .
$$

This implies the following estimate for the jump of $v \in \bigoplus_{T \in \mathcal{T}_{K}} H^{1}(T)$ :

$$
\left\|\llbracket v \rrbracket_{F}\right\|_{F}^{2} \leq C h_{K}^{-1}\|v\|_{K}^{2}+C h_{K} \sum_{T \in \mathcal{T}_{K}}|v|_{1, T}^{2} \quad \forall F \in \mathcal{F}_{K}
$$


and for the jump term $j_{K}(\cdot, \cdot)$ we get for all $H^{1}(K) \cap\left(\bigoplus_{T \in \mathcal{T}_{K}} H^{2}(T)\right)$

$$
j_{K}(v, v) \leq C \gamma_{0} \beta_{\infty} h_{K}\left\{|v|_{1, K}^{2}+h_{K}^{2} \sum_{T \in \mathcal{T}_{K}}|v|_{2, T}^{2}\right\} .
$$

Applying (3.13), (3.11), and inverse inequalities, we infer for all $K \in \mathcal{M}_{h}$ the estimate

$$
\left\|v_{h}\right\|_{K} \leq\left\|v_{h}\right\|_{S, K} \leq C h_{K}^{-1 / 2}\left\|v_{h}\right\|_{K} \quad \forall v_{h} \in V_{h} .
$$

3.3.2. Stability of the method. From partial integration of the convective term we get for all $w_{h} \in V_{h}$

$$
a_{h}\left(w_{h}, w_{h}\right)=\left(\sigma-\frac{1}{2} \operatorname{div} \beta, w_{h}^{2}\right)_{\Omega}+\frac{1}{2}\left\langle|\beta \cdot n|, w_{h}^{2}\right\rangle_{\Gamma}+\sum_{K \in \mathcal{M}_{h}} j_{K}\left(w_{h}, w_{h}\right)
$$

which implies the coercivity of $a_{h}(\cdot, \cdot)$ :

$$
a_{h}\left(w_{h}, w_{h}\right) \geq\left\|w_{h}\right\|^{2} \quad \forall w_{h} \in V_{h} .
$$

Our aim is now to prove the following improved stability estimate in the strong norm.

Lemma 3.1. Assume that the mesh $\mathcal{T}_{h}$ is shape regular such that the standard inverse inequalities as well as the condition (2.1) hold. Let the parameter $\gamma_{0}$ in the definition (3.6) of $j_{K}(\cdot, \cdot)$ satisfy $\gamma_{0} \geq C\|\beta\|_{L^{\infty}(\Omega)^{d}}$. Then, there exists an $h$-independent constant $\alpha_{0}>0$ that satisfies the stability estimate

$$
\alpha_{0}\left\|w_{h}\right\|_{S} \leq \sup _{v_{h} \in V_{h} \backslash\{0\}} \frac{a_{h}\left(w_{h}, v_{h}\right)}{\left\|v_{h}\right\|_{S}} \quad \forall w_{h} \in V_{h} .
$$

Proof. The idea of the proof is to construct, for a given $w_{h} \in V_{h}$, an associated test function $v_{h}$ in the form $v_{h}=c w_{h}+z_{h}$ with a sufficiently large constant $c>0$ such that $a_{h}\left(w_{h}, v_{h}\right)$ contains the "good" term $a_{h}\left(w_{h}, c w_{h}\right) \geq c\left\|w_{h}\right\|^{2}$ which can be used to control "bad" terms coming from the estimate for $a_{h}\left(w_{h}, z_{h}\right)$. The role of $z_{h}$ is only to act as a "test partner" for the convective term such that $\left(\beta \cdot \nabla w_{h}, z_{h}\right)_{\Omega}$ will produce on each composite cell $K \in \mathcal{M}_{h}$ the good term $h_{K}\left\|\beta \cdot \nabla w_{h}\right\|_{K}^{2}$ with possibly some bad terms that can be controlled.

In the following, we will construct $z_{h} \in V_{h}$ separately on each cell $K \in \mathcal{M}_{h}$ with the property that $\left.z_{h}\right|_{K}$ vanishes at the boundary of $K$. Therefore, the pieces $\left.z_{h}\right|_{K} \in$ $U_{r}(K)$ fit together into a global function in $V_{h}$. With the constant approximation $\beta_{K}:=\beta\left(c_{K}\right)$ used in the definition $(3.6)$ of $j_{K}(\cdot, \cdot)$ we define

$$
\left.z_{h}\right|_{K}:=h_{K} I_{\mathrm{av}}^{K, r}\left(\left(\beta_{K} \cdot \nabla w_{h}\right) \xi_{K}\right) \in U_{r}(K) .
$$

For a a more compact notation, we introduce the following abbreviations,

$$
w_{\beta}:=\beta \cdot \nabla w_{h}, \quad w_{\beta}^{K}:=\beta_{K} \cdot \nabla w_{h}, \quad \text { and } \quad \beta_{1, \infty}:=|\beta|_{W^{1, \infty}(\Omega)} .
$$

Using the estimate $\left\|\beta-\beta_{K}\right\|_{L^{\infty}(K)} \leq h_{K} \beta_{1, \infty}$ and an inverse inequality we get

$$
\left(w_{\beta}, z_{h}\right)_{K} \geq\left(w_{\beta}^{K}, z_{h}\right)_{K}-C \beta_{1, \infty}\left\|w_{h}\right\|_{K}\left\|z_{h}\right\|_{K} .
$$

Copyright (c) by SIAM. Unauthorized reproduction of this article is prohibited. 
From (2.3), (3.12), and an inverse inequality we infer with $C_{2}=C_{1}\left(r-1, \frac{1}{2}\right)$

$$
\begin{aligned}
\left(w_{\beta}^{K}, z_{h}\right)_{K} & =h_{K}\left(w_{\beta}^{K}, w_{\beta}^{K} \xi_{K}\right)_{K}+h_{K}\left(w_{\beta}^{K}, I_{\mathrm{av}}^{K, r}\left(w_{\beta}^{K} \xi_{K}\right)-w_{\beta}^{K} \xi_{K}\right)_{K} \\
& \geq C_{2} h_{K}\left\|w_{\beta}^{K}\right\|_{K}^{2}-C h_{K}^{3 / 2}\left\|w_{\beta}^{K}\right\|_{K} \sum_{F \in \mathcal{F}_{K}}\left\|\llbracket w_{\beta}^{K} \xi_{K} \rrbracket_{F}\right\|_{F} .
\end{aligned}
$$

Since the space $V_{h}$ is conforming, all tangential derivatives of $w_{h} \in V_{h}$ with respect to a face $F \in \mathcal{F}_{K}$ do not have a jump across $F$. Therefore, we have $\llbracket \nabla w_{h} \rrbracket_{F}=\llbracket \frac{\partial w_{h}}{\partial n_{F}} \rrbracket_{F} n_{F}$ which implies, due to the continuity of $\xi_{K}$, that

$$
\llbracket w_{\beta}^{K} \xi_{K} \rrbracket_{F}=\llbracket\left(\beta_{K} \cdot \nabla w_{h}\right) \xi_{K} \rrbracket_{F}=\left(\left(\beta_{K} \cdot n_{F}\right) \llbracket \frac{\partial w_{h}}{\partial n_{F}} \rrbracket_{F}\right) \xi_{K}
$$

and finally, using $0 \leq \xi_{K} \leq 1$, for all $F \in \mathcal{F}_{K}$

$$
\left\|\llbracket w_{\beta}^{K} \xi_{K} \rrbracket_{F}\right\|_{F} \leq\left|\beta_{K} \cdot n_{F}\right|\left\|\llbracket \nabla w_{h} \rrbracket_{F}\right\|_{F} \leq h_{K}^{-1}\left\{\frac{\beta_{\infty}}{\gamma_{0}} j_{K}\left(w_{h}, w_{h}\right)\right\}^{1 / 2} .
$$

From (3.19) we get by means of Young's inequality

$$
\left(w_{\beta}^{K}, z_{h}\right)_{K} \geq C_{2} h_{K}\left\|w_{\beta}^{K}\right\|_{K}^{2}-C h_{K}^{1 / 2}\left\|w_{\beta}^{K}\right\|_{K}\left\|w_{h}\right\|_{K} \geq \frac{C_{2}}{2} h_{K}\left\|w_{\beta}^{K}\right\|_{K}^{2}-C\left\|w_{h}\right\|_{K}^{2} .
$$

Using $\left\|w_{\beta}\right\|_{K}^{2} \leq 2\left\|w_{\beta}^{K}\right\|_{K}^{2}+2\left\|w_{\beta}-w_{\beta}^{K}\right\|_{K}^{2}$ we obtain with an inverse inequality

$$
\left\|w_{\beta}^{K}\right\|_{K}^{2} \geq \frac{1}{2}\left\|w_{\beta}\right\|_{K}^{2}-C \beta_{1, \infty}^{2}\left\|w_{h}\right\|_{K}^{2}
$$

which implies

$$
\left(w_{\beta}^{K}, z_{h}\right)_{K} \geq \frac{C_{2}}{4} h_{K}\left\|w_{\beta}\right\|_{K}^{2}-C\left\|w_{h}\right\|_{K}^{2} .
$$

This together with (3.18) yields

$$
\begin{aligned}
& a_{h}\left(w_{h}, z_{h}\right)=\sum_{K \in \mathcal{M}_{h}}\left\{\left(w_{\beta}, z_{h}\right)_{K}+\left(\sigma w_{h}, z_{h}\right)_{K}+j_{K}\left(w_{h}, z_{h}\right)+\left\langle\left|\beta \cdot n_{K}\right| w_{h}, z_{h}\right\rangle_{\partial K \cap \Gamma^{-}}\right\} \\
& (3.21) \geq \sum_{K \in \mathcal{M}_{h}}\left\{\frac{C_{2}}{4} h_{K}\left\|w_{\beta}\right\|_{K}^{2}-C\left\|w_{h}\right\|_{K}^{2}-C\left\|w_{h}\right\|_{K}\left\|z_{h}\right\|_{K}\right\} .
\end{aligned}
$$

In the following, we will derive an upper bound for $\left\|z_{h}\right\|_{K}$. Using (2.4) and an inverse inequality we get

$$
\begin{aligned}
\left\|z_{h}\right\|_{K} & =h_{K}\left\|I_{\mathrm{av}}^{K, r}\left(w_{\beta}^{K} \xi_{K}\right)\right\|_{K} \leq C h_{K}\left\|w_{\beta}^{K} \xi_{K}\right\|_{K} \leq C h_{K}\left\|w_{\beta}^{K}\right\|_{K} \\
& \leq C h_{K}\left\|w_{\beta}\right\|_{K}+C h_{K} \beta_{1, \infty}\left\|w_{h}\right\|_{K},
\end{aligned}
$$

which implies with (3.14) that

$$
\left\|z_{h}\right\|_{K} \leq\left\|z_{h}\right\|_{S, K} \leq C h_{K}^{1 / 2}\left\|w_{\beta}\right\|_{K}+C h_{K}^{1 / 2}\left\|w_{h}\right\|_{K} .
$$

We apply this estimate for $\left\|z_{h}\right\|_{K}$ in (3.21) and obtain by means of Young's inequality

$$
\begin{aligned}
a_{h}\left(w_{h}, z_{h}\right) & \geq \sum_{K \in \mathcal{M}_{h}}\left\{\frac{C_{2}}{4} h_{K}\left\|w_{\beta}\right\|_{K}^{2}-C\left\|w_{h}\right\|_{K} h_{K}^{1 / 2}\left\|w_{\beta}\right\|_{K}-C\left\|w_{h}\right\|_{K}^{2}\right\} \\
& \geq \sum_{K \in \mathcal{M}_{h}}\left\{\frac{C_{2}}{8} h_{K}\left\|w_{\beta}\right\|_{K}^{2}-C\left\|w_{h}\right\|_{K}^{2}\right\} .
\end{aligned}
$$

Copyright $@$ by SIAM. Unauthorized reproduction of this article is prohibited. 
Thus, we have shown that there are $h$-independent constants $C_{3}:=C_{2} / 8$ and $C_{4}>0$ such that

$$
a_{h}\left(w_{h}, z_{h}\right) \geq C_{3}\left\|w_{h}\right\|_{S}^{2}-C_{4}\left\|w_{h}\right\|^{2} .
$$

Now, with these preparations it is easy to prove our stability result. The estimate (3.16) is obvious in the case $w_{h}=0$. Let $w_{h}$ be an arbitrary element of $V_{h} \backslash\{0\}$ and $z_{h} \in V_{h}$ the elementwise defined function from (3.17). Taking the sum of the squares of the $K$-local estimates (3.22) we infer that there exists an $h$ independent constant $C_{5}>0$ such that $\left\|z_{h}\right\|_{S} \leq C_{5}\left\|w_{h}\right\|_{S}$. Then, we get for the function $v_{h}:=\left(C_{4}+C_{5}\right) w_{h}+z_{h}$ by means of (3.15) and (3.23)

$$
a_{h}\left(w_{h}, v_{h}\right)=\left(C_{4}+C_{5}\right) a_{h}\left(w_{h}, w_{h}\right)+a_{h}\left(w_{h}, z_{h}\right) \geq C_{3}\left\|w_{h}\right\|_{S}^{2} .
$$

Therefore, we get from

$$
\left\|v_{h}\right\|_{S} \leq\left(C_{4}+C_{5}\right)\left\|w_{h}\right\|_{S}+\left\|z_{h}\right\|_{S} \leq\left(C_{4}+2 C_{5}\right)\left\|w_{h}\right\|_{S}
$$

the final estimate $a_{h}\left(w_{h}, v_{h}\right) \geq C_{3}\left\|w_{h}\right\|_{S}^{2} \geq \alpha_{0}\left\|w_{h}\right\|_{S}\left\|v_{h}\right\|_{S}$ with $\alpha_{0}=C_{3} /\left(C_{4}+2 C_{5}\right)$ which proves (3.16). Note that the property $v_{h} \neq 0$ follows from $w_{h} \neq 0$ and

$$
\left\|v_{h}\right\|_{S} \geq\left(C_{4}+C_{5}\right)\left\|w_{h}\right\|_{S}-\left\|z_{h}\right\|_{S} \geq C_{4}\left\|w_{h}\right\|_{S}>0 .
$$

3.3.3. Estimates for the interpolation error. Let $i_{h}: H^{r+1}(\Omega) \rightarrow V_{h}$ denote the standard finite element interpolation operator satisfying the optimal local approximation properties for $s \in\{0,1,2\}$,

$$
\sum_{T \in \mathcal{T}_{K}}\left|u-i_{h} u\right|_{s, T}^{2} \leq C h_{K}^{2(r+1-s)}|u|_{r+1, K}^{2} \quad \forall K \in \mathcal{M}_{h}
$$

In the following we will prove a local interpolation error estimate in the norm $\|\cdot\|_{S, K}$.

LEMma 3.2. Let $r \geq 1$ be the order of the finite element space $V_{h}$ defined in (3.4) and $i_{h} u \in V_{h}$ the interpolate of $u \in H^{r+1}(\Omega)$. Then, it holds the estimate

$$
\left\|u-i_{h} u\right\|_{S, K} \leq C h_{K}^{r+1 / 2}|u|_{r+1, K} \quad \forall K \in \mathcal{M}_{h} .
$$

Proof. Let $\eta:=u-i_{h} u$ and $K \in \mathcal{M}_{h}$ be an arbitrary composite element. Then, we have that $\left.\eta\right|_{K} \in H^{2}(K)+D_{r}(K)$, and (3.13) together with (3.24) yield

$$
j_{K}(\eta, \eta) \leq C \gamma_{0} \beta_{\infty} h_{K}\left\{|\eta|_{1, K}^{2}+h_{K}^{2} \sum_{T \in \mathcal{T}_{K}}|\eta|_{2, T}^{2}\right\} \leq C h_{K}^{2 r+1}|u|_{r+1, K}^{2} .
$$

Applying the estimate (3.11) for each element face $F \subset \partial K \cap \Gamma$ we get

$$
\left\||\beta \cdot n|^{1 / 2} \eta\right\|_{\partial K \cap \Gamma}^{2} \leq C \beta_{\infty} h_{K}^{-1}\left\{\|\eta\|_{K}^{2}+h_{K}^{2} \sum_{T \in \mathcal{T}_{K}}|\eta|_{1, T}^{2}\right\} \leq C h_{K}^{2 r+1}|u|_{r+1, K}^{2} .
$$

Thus, the estimate $h_{K}\|\beta \cdot \nabla \eta\|_{K}^{2} \leq C \beta_{\infty}^{2} h_{K}|\eta|_{1, K}^{2}$, the $L^{2}$-norm estimate of $\eta$, (3.26), and (3.27) imply the assertion (3.25).

Finally, we will prove an estimate of the interpolation error in terms of the bilinear form $a_{h}(\cdot, \cdot)$.

Copyright $@$ by SIAM. Unauthorized reproduction of this article is prohibited. 
Lemma 3.3. Assume the setting of Lemma 3.2. Then, it holds the estimate

$$
\left|a_{h}\left(u-i_{h} u, v_{h}\right)\right| \leq C\left\{\sum_{K \in \mathcal{M}_{h}} h_{K}^{2 r+1}|u|_{r+1, K}^{2}\right\}^{1 / 2}\left\|v_{h}\right\|_{S} \quad \forall v_{h} \in V_{h} .
$$

Proof. Using the notation $\eta:=u-i_{h} u$ we have that $\eta \in H^{1}(\Omega)$ such that partial integration of the term $\left(\beta \cdot \nabla \eta, v_{h}\right)_{\Omega}$ gives

$a_{h}\left(\eta, v_{h}\right)=-\left(\eta, \beta \cdot \nabla v_{h}\right)_{\Omega}+\left(\sigma-\operatorname{div} \beta, \eta v_{h}\right)_{\Omega}+\left\langle(\beta \cdot n), \eta v_{h}\right\rangle_{\Gamma \backslash \Gamma^{-}}+\sum_{K \in \mathcal{M}_{h}} j_{K}\left(\eta, v_{h}\right)$.

Now, we apply the estimates $j_{K}\left(\eta, v_{h}\right) \leq\|\eta\|_{K}\left\|v_{h}\right\|_{K}$ and $\|\sigma-\operatorname{div} \beta\|_{L^{\infty}(\Omega)} \leq C$ to obtain

$$
\begin{aligned}
\left|a_{h}\left(\eta, v_{h}\right)\right| & \leq \sum_{K \in \mathcal{M}_{h}}\left\{h_{K}^{-1 / 2}\|\eta\|_{K}+C\|\eta\|_{K}+\left.C\|\| \beta \cdot n\right|^{1 / 2} \eta\left\|_{\partial K \cap\left(\Gamma \backslash \Gamma^{-}\right)}+\right\| \eta \|_{K}\right\}\left\|v_{h}\right\|_{S, K} \\
& \leq \sum_{K \in \mathcal{M}_{h}}\left\{h_{K}^{-1 / 2}\|\eta\|_{K}+C\|\eta\|_{K}\right\}\left\|v_{h}\right\|_{S, K} .
\end{aligned}
$$

The assertion (3.28) follows from the Cauchy-Schwarz inequality and Lemma 3.2.

3.3.4. Final error estimate. Now, we are ready to prove our main result on the discretization error of the local CIP discretization.

THEOREM 3.4. Let $u_{h} \in V_{h}$ denote the solution of the discrete problem (3.7) and $u \in V$ the solution of the continuous problem (3.3) which is supposed to have the regularity $u \in H^{r+1}(\Omega)$ with $r \geq 1$ denoting the polynomial order of $V_{h}$. Assume that the mesh $\mathcal{T}_{h}$ is shape regular such that the standard inverse inequalities as well as the condition (2.1) hold. Let the parameter $\gamma_{0}$ in the definition (3.6) of $j_{K}(\cdot, \cdot)$ satisfy $\gamma_{0} \geq C\|\beta\|_{L^{\infty}(\Omega)^{d}}$ and assume that the data $\beta$ and $\sigma$ satisfy (3.2). Then, there exists a unique solution $u_{h}$ of the discrete problem (3.7) and there is an h-independent constant $C>0$ satisfying the error estimate

$$
\left\|u-u_{h}\right\|_{S} \leq C\left\{\sum_{K \in \mathcal{M}_{h}} h_{K}^{2 r+1}|u|_{r+1, K}^{2}\right\}^{1 / 2} .
$$

Proof. From the coercivity (3.15) of the bilinear form $a_{h}$ the uniqueness of the discrete solution follows. Since the discrete problem is equivalent to a linear system of equations for the nodal values, the existence of the discrete solution follows from its uniqueness. To prove the error estimate we split the error as

$$
u-u_{h}=\left(u-i_{h} u\right)+\left(i_{h} u-u_{h}\right)=: \eta+e_{h}
$$

into the interpolation error $\eta$ and the discrete error $e_{h}=i_{h} u-u_{h} \in V_{h}$. From the regularity assumption we have at least that $u \in H^{2}(\Omega)$. Therefore, the jumps $\llbracket \nabla u \rrbracket_{F}$ vanish for all faces $F$ of the mesh such that $j_{K}\left(u, v_{h}\right)=0$ for all $v_{h} \in V_{h}$ and $K \in \mathcal{M}_{h}$. This implies the consistency of our method, i.e.,

$$
a_{h}\left(u-u_{h}, v_{h}\right)=0 \quad \forall v_{h} \in V_{h} .
$$

By means of the stability estimate (3.16) applied to $w_{h}=e_{h}$ there exists a $v_{h} \in V_{h} \backslash\{0\}$ such that

$$
\alpha_{0}\left\|e_{h}\right\|_{S}\left\|v_{h}\right\|_{S} \leq a_{h}\left(e_{h}, v_{h}\right)=a_{h}\left(u-u_{h}, v_{h}\right)-a_{h}\left(\eta, v_{h}\right) .=-a_{h}\left(\eta, v_{h}\right) .
$$

Copyright $@$ by SIAM. Unauthorized reproduction of this article is prohibited. 
Applying Lemma 3.3 and dividing by $\left\|v_{h}\right\|_{S} \neq 0$ we infer

$$
\left\|e_{h}\right\|_{S} \leq C \alpha_{0}^{-1}\left\{\sum_{K \in \mathcal{M}_{h}} h_{K}^{2 r+1}|u|_{r+1, K}^{2}\right\}^{1 / 2} .
$$

For the interpolation error $\eta$, we get from Lemma 3.2

$$
\|\eta\|_{S}^{2}=\sum_{K \in \mathcal{M}_{h}}\|\eta\|_{S, K}^{2} \leq C \sum_{K \in \mathcal{M}_{h}} h_{K}^{2 r+1}|u|_{r+1, K}^{2}
$$

Finally, the estimate (3.29) follows by means of the triangle inequality.

\section{Local CIP discretization for the Stokes-Brinkman problem.}

4.1. The Stokes-Brinkman problem. Let again $\Omega \subset \mathbb{R}^{d}, d \leq 3$, denote an open, bounded, and connected domain with a polygonal boundary $\Gamma, f \in L^{2}(\Omega)^{d}$ a given vector field, and $g \in L^{2}(\Omega)$ a given scalar source field satisfying $(g, 1)_{\Omega}=0$. Then we consider the model problem: Find the velocity $u: \Omega \rightarrow \mathbb{R}^{d}$ and the pressure $p: \Omega \rightarrow \mathbb{R}$ such that

$$
\begin{array}{rlr}
-\nu \Delta u+\sigma u+\nabla p=f & & \text { in } \Omega, \\
\operatorname{div} u=g & & \text { in } \Omega, \\
u=0 & & \text { on } \Gamma,
\end{array}
$$

where $\nu>0$ denotes the constant kinematic viscosity and $\sigma \geq 0$ another given constant parameter. We introduce the weak solution spaces $X:=\left(H_{0}^{1}(\Omega)\right)^{d}$ and $M:=L_{0}^{2}(\Omega)=\left\{q \in L^{2}(\Omega):(q, 1)_{\Omega}=0\right\}$ for velocity and pressure, respectively, and the bilinear forms $a(\cdot, \cdot)$ and $b(\cdot, \cdot)$ defined by

$$
a(u, v):=\nu(\nabla u, \nabla v)_{\Omega}+\sigma(u, v)_{\Omega}, \quad b(v, p):=-(\operatorname{div} v, p)_{\Omega},
$$

where $\nabla u$ denotes the gradient tensor and $(\cdot, \cdot)_{\Omega}$ the inner product in $L^{2}(\Omega)^{d \times d}$, $L^{2}(\Omega)^{d}$, and $L^{2}(\Omega)$, depending on the context. For a more compact notation, we define the product space $Y:=X \times M$ and the bilinear form $A: Y \times Y \rightarrow \mathbb{R}$ by means of

$$
A((u, p),(v, q)):=a(u, v)+b(v, p)-b(u, q) .
$$

Then, the weak formulation of the Stokes-Brinkman problem reads as follows: Find $(u, p) \in X \times M$ such that

$$
A((u, p),(v, q))=(f, v)_{\Omega}+(g, q)_{\Omega} \quad \forall(v, q) \in X \times M .
$$

4.2. Local CIP discretization. For the velocity approximation, we choose a continuous finite element space $X_{h} \subset X$ defined as

$$
X_{h}:=\left\{v \in X:\left.v\right|_{T} \in \mathbb{P}_{r}(T)^{d} \quad \forall T \in \mathcal{T}_{K}, K \in \mathcal{M}_{h}\right\},
$$

where $r$ again denotes the polynomial order of $X_{h}$. Note that, for our error analysis, we will need the assumption $r \geq 2$ in order to construct a special interpolation operator $\widetilde{I}_{h}^{r}$; see section 4.3.3. The pressure will be approximated by means of discontinuous functions from the space

$$
Q_{h}:=\left\{q \in L^{2}(\Omega):\left.q\right|_{K} \in U_{m}(K) \quad \forall K \in \mathcal{M}_{h}\right\}, \quad 1 \leq m \leq r,
$$


where $m$ denotes the polynomial order of the pressure approximation and $U_{m}(K)$ the continuous composite space on the macroelement $K$ defined in (2.2). The solution space for the discrete pressure is defined as $M_{h}:=Q_{h} \cap M$. Note that with the above definition a discrete pressure function $q_{h} \in Q_{h}$ is continuous on each macroelement.

It is well known that, in general, such a choice of the spaces $X_{h}$ and $M_{h}$ does not satisfy the inf-sup condition uniformly with respect to $h$. Therefore, we apply the local CIP method as a pressure stabilization and add, for each macroelement $K \in \mathcal{M}_{h}$, the following jump terms of the pressure gradients,

$$
J_{K}(p, q):=\delta_{K} h_{K} \sum_{F \in \mathcal{F}_{K}}\left\langle\llbracket \nabla p \rrbracket_{F}, \llbracket \nabla q \rrbracket_{F}\right\rangle_{F}, \quad \delta_{K}:=\min \left(\frac{h_{K}^{2}}{\nu}, h_{K}\right) .
$$

The bilinear form $A(\cdot, \cdot)$ is modified by the discrete bilinear form $A_{h}: Y_{h} \times Y_{h} \rightarrow \mathbb{R}$ on the discrete space $Y_{h}:=X_{h} \times M_{h}$ as follows:

$$
\begin{aligned}
A_{h}\left(\left(u_{h}, p_{h}\right),\left(v_{h}, q_{h}\right)\right):= & A\left(\left(u_{h}, p_{h}\right),\left(v_{h}, q_{h}\right)\right) \\
& +\sum_{K \in \mathcal{M}_{h}}\left\{J_{K}\left(p_{h}, q_{h}\right)+h_{K}\left(\operatorname{div} u_{h}, \operatorname{div} v_{h}\right)_{K}\right\}
\end{aligned}
$$

and the associated discrete form on the right-hand side becomes

$$
\ell_{h}\left(\left(v_{h}, q_{h}\right)\right):=\left(f, v_{h}\right)_{\Omega}+\left(g, q_{h}\right)_{\Omega}+\sum_{K \in \mathcal{M}_{h}} h_{K}\left(g, \operatorname{div} v_{h}\right)_{K} .
$$

Now, the discrete problem reads as follows: Find $\left(u_{h}, p_{h}\right) \in X_{h} \times M_{h}$ such that

$$
A_{h}\left(\left(u_{h}, p_{h}\right),\left(v_{h}, q_{h}\right)\right)=\ell_{h}\left(\left(v_{h}, q_{h}\right)\right) \quad \forall\left(v_{h}, q_{h}\right) \in X_{h} \times M_{h} .
$$

This formulation is similar to that proposed in [3] using local projection stabilization. Note that this local CIP version again has the advantage compared to the classical CIP version that a static condensation of all dofs in the interior of each composite cell, except for the pressure constant, is possible.

\subsection{Error analysis.}

4.3.1. Discrete norms and seminorms. For a function pair $(v, q) \in\left(X+X_{h}\right) \times$ $\left(H^{2}(\Omega)+Q_{h}\right)$ and an arbitrary composite element $K \in \mathcal{M}_{h}$, we introduce the local seminorms

$$
\|(v, q)\|_{K}^{2}:=\nu|v|_{1, K}^{2}+\sigma\|v\|_{K}^{2}+h_{K}\|\operatorname{div} v\|_{K}^{2}+J_{K}(q, q)
$$

and

$$
\|(v, q)\|_{S, K}^{2}:=\|(v, q)\|_{K}^{2}+\delta_{K}\|\nabla q\|_{K}^{2},
$$

where the symbol $S$ indicates a strong seminorm. To these local semi-norms we assign the corresponding global ones

$$
\|(v, q)\|^{2}:=\sum_{K \in \mathcal{M}_{h}}\|(v, q)\|_{K}^{2} \quad \text { and } \quad\|(v, q)\|_{S}^{2}:=\sum_{K \in \mathcal{M}_{h}}\|(v, q)\|_{S, K}^{2} .
$$

Using the estimate $(3.12)$ we infer for the jump term $J_{K}(\cdot, \cdot)$ defined in $(4.6)$ that

$$
J_{K}(q, q) \leq C \delta_{K}\left\{|q|_{1, K}^{2}+h_{K}^{2} \sum_{T \in \mathcal{T}_{K}}|q|_{2, T}^{2}\right\} \quad \forall q \in H^{2}(K)+D_{m}(K),
$$

Copyright (C) by SIAM. Unauthorized reproduction of this article is prohibited. 
which yields that by means of inverse inequalities for all $K \in \mathcal{M}_{h}$ and all $\left(v_{h}, q_{h}\right) \in$ $X_{h} \times Q_{h}$ it holds

$$
\left\|\left(v_{h}, q_{h}\right)\right\|_{S, K}^{2} \leq C\left\{\nu\left|v_{h}\right|_{1, K}^{2}+\sigma\|v\|_{K}^{2}+h_{K}\left\|\operatorname{div} v_{h}\right\|_{K}^{2}+\delta_{K}\left\|\nabla q_{h}\right\|_{K}^{2}\right\} .
$$

4.3.2. Stability of the method. From the definition of $A_{h}(\cdot, \cdot)$ we obtain the following partial coercivity of $A_{h}$ with respect to the seminorm $\|\cdot\|$ :

$$
A_{h}\left(\left(w_{h}, q_{h}\right),\left(w_{h}, q_{h}\right)\right) \geq\left\|\left(w_{h}, q_{h}\right)\right\|^{2} \quad \forall\left(w_{h}, q_{h}\right) \in X_{h} \times Q_{h} .
$$

Our aim is now to prove the following improved stability estimate in the strong seminorm.

Lemma 4.1. Assume that the mesh $\mathcal{T}_{h}$ is shape regular such that the standard inverse inequalities as well as the condition (2.1) hold. Assume $1 \leq m \leq r$ for the polynomial orders $r$ and $m$ of the finite element spaces $X_{h}$ and $Q_{h}$, respectively. Furthermore, suppose that the parameter $\sigma$ in problem (4.1) satisfies $\sigma \leq C h^{-1}$ with an $h$-independent positive constant $C$. Then, there exists an $h$-independent constant $\alpha_{1}>0$ that satisfies the following stability property of the bilinear form $A_{h}$ on the product space $Y_{h}:=X_{h} \times M_{h}$ :

$$
\forall \phi_{h} \in Y_{h} \quad \exists \theta_{h} \in Y_{h}: \quad \alpha_{1}\left\|\phi_{h}\right\|_{S}^{2} \leq A_{h}\left(\phi_{h}, \theta_{h}\right) \quad \text { and } \quad\left\|\theta_{h}\right\|_{S} \leq\left\|\phi_{h}\right\|_{S} .
$$

Proof. At first, we will prove that there are h-independent constants $\alpha, C>0$ such that for all $\left(w_{h}, q_{h}\right) \in Y_{h}=X_{h} \times M_{h}$ there exists an element $\left(v_{h}, s_{h}\right) \in Y_{h}$ satisfying

$$
\alpha\left\|\left(w_{h}, q_{h}\right)\right\|_{S}^{2} \leq A_{h}\left(\left(w_{h}, q_{h}\right),\left(v_{h}, s_{h}\right)\right) \quad \text { and } \quad\left\|\left(v_{h}, s_{h}\right)\right\|_{S} \leq C\left\|\left(w_{h}, q_{h}\right)\right\|_{S} .
$$

The idea is to construct, similarly to section 3.3 .2 , for a given $\left(w_{h}, q_{h}\right) \in Y_{h}$, an associated test function $\left(v_{h}, s_{h}\right) \in Y_{h}$ in the form $\left(v_{h}, s_{h}\right)=c\left(w_{h}, q_{h}\right)+\left(z_{h}, 0\right)$ with a sufficiently large constant $c>0$ where the role of $z_{h}$ is to act as a "test partner" in the term $b\left(z_{h}, q_{h}\right)$ in order to produce the good term $h_{K}^{2}\left\|\nabla q_{h}\right\|_{K}^{2}$ on each composite cell $K \in \mathcal{M}_{h}$.

In the following, we will construct $z_{h} \in X_{h}$ separately on each cell $K \in \mathcal{M}_{h}$ with the property that $\left.z_{h}\right|_{K}$ vanishes at the boundary of $K$. Therefore, the pieces $\left.z_{h}\right|_{K} \in\left(U_{r}(K)\right)^{d}$ fit together into a global function in $X_{h}$. We define for the given $q_{h} \in M_{h}$

$$
\left.z_{h}\right|_{K}:=\delta_{K} I_{\mathrm{av}}^{K, m}\left(\left(\left.\nabla q_{h}\right|_{K}\right) \xi_{K}\right) \in U_{m}(K)^{d},
$$

where $\xi_{K} \in U_{1}(K)$ is the same scalar bubble function as in section 2.3 and the vectorvalued averaging operator $I_{\mathrm{av}}^{K, m}: D_{m}(K)^{d} \rightarrow U_{m}(K)^{d}$ is defined by applying the scalar version of $I_{\mathrm{av}}^{K, m}$ defined in section 2.2 to each component of the vector function $\left(\left.\nabla q_{h}\right|_{K}\right) \xi_{K} \in D_{m}(K)^{d}$.

Now, for the $K$-local part of $b\left(z_{h}, q_{h}\right)$ we obtain by means of partial integration, $\left.z_{h}\right|_{K} \in H_{0}^{1}(K)^{d}$, and (2.3),

$$
\begin{aligned}
-\left(\operatorname{div} z_{h}, q_{h}\right)_{K} & =\left(z_{h}, \nabla q_{h}\right)_{K} \\
& =\delta_{K}\left\|\xi_{K}^{1 / 2} \nabla q_{h}\right\|_{K}^{2}+\delta_{K}\left(I_{\mathrm{av}}^{K, m}\left(\xi_{K} \nabla q_{h}\right)-\xi_{K} \nabla q_{h}, \nabla q_{h}\right)_{K} \\
& \geq C_{2} \delta_{K}\left\|\nabla q_{h}\right\|_{K}^{2}-C \delta_{K} h_{K}^{1 / 2}\left\|\nabla q_{h}\right\|_{K} \sum_{F \in \mathcal{F}_{K}}\left\|\llbracket \xi_{K} \nabla q_{h} \rrbracket_{F}\right\|_{F} .
\end{aligned}
$$

Copyright $@$ by SIAM. Unauthorized reproduction of this article is prohibited. 
Since $\xi_{K}$ is continuous on $K$ and $0 \leq \xi_{K} \leq 1$, we have for all $F \in \mathcal{F}_{K}$

$$
\left\|\llbracket \xi_{K} \nabla q_{h} \rrbracket_{F}\right\|_{F} \leq\left\|\llbracket \nabla q_{h} \rrbracket_{F}\right\|_{F} \leq \delta_{K}^{-1 / 2} h_{K}^{-1 / 2}\left\{J_{K}\left(q_{h}, q_{h}\right)\right\}^{1 / 2}
$$

which implies with (4.18) by means of Young's inequality

$$
\begin{aligned}
-\left(\operatorname{div} z_{h}, q_{h}\right)_{K} & \geq \frac{C_{2}}{2} \delta_{K}\left\|\nabla q_{h}\right\|_{K}^{2}-C J_{K}\left(q_{h}, q_{h}\right) \\
& \geq \frac{C_{2}}{2}\left\|\left(w_{h}, q_{h}\right)\right\|_{S, K}^{2}-C^{\prime}\left\|\left(w_{h}, q_{h}\right)\right\|_{K}^{2} .
\end{aligned}
$$

For a more compact notation, we define $\phi_{h}, \psi_{h} \in Y_{h}$ by

$$
\phi_{h}:=\left(w_{h}, q_{h}\right), \quad \psi_{h}:=\left(z_{h}, 0\right) .
$$

Then we get from the definition (4.7) of $A_{h}$ and (4.19)

$$
\begin{aligned}
A_{h}\left(\phi_{h}, \psi_{h}\right) & =a\left(w_{h}, z_{h}\right)+\sum_{K \in \mathcal{M}_{h}}\left\{-\left(\operatorname{div} z_{h}, q_{h}\right)_{K}+h_{K}\left(\operatorname{div} w_{h}, \operatorname{div} z_{h}\right)_{K}\right\} \\
& \geq \sum_{K \in \mathcal{M}_{h}}\left\{\frac{C_{2}}{2}\left\|\phi_{h}\right\|_{S, K}^{2}-C^{\prime}\left\|\phi_{h}\right\|_{K}^{2}-\left\|\phi_{h}\right\|_{K}\left\|\psi_{h}\right\|_{K}\right\} .
\end{aligned}
$$

In the following, we will derive an upper bound for $\left\|\psi_{h}\right\|_{K}$ under the assumption that $\sigma \leq C h^{-1}$. Using (4.17), (2.4), $\left|\xi_{K}\right| \leq 1$, an inverse inequality, the bound $\sigma \leq C h_{K}^{-1}$, and the property $\delta_{K}\left(\nu+h_{K}\right) \leq 2 h_{K}^{2}$ we get

$$
\begin{aligned}
\left\|\psi_{h}\right\|_{S, K}^{2} & \leq\left(\nu+h_{K}\right)\left|z_{h}\right|_{1, K}^{2}+\sigma\left\|z_{h}\right\|_{K}^{2} \\
& \leq C\left(\nu+h_{K}+\sigma h_{K}^{2}\right) h_{K}^{-2} \delta_{K}^{2}\left\|I_{\mathrm{av}}^{K, m}\left(\xi_{K} \nabla q_{h}\right)\right\|_{K}^{2} \\
& \leq C\left(\nu+h_{K}\right) h_{K}^{-2} \delta_{K}^{2}\left\|\nabla q_{h}\right\|_{K}^{2} \leq C\left\|\phi_{h}\right\|_{S, K}^{2},
\end{aligned}
$$

which implies with (4.11) that

$$
\left\|\psi_{h}\right\|_{K} \leq\left\|\psi_{h}\right\|_{S, K} \leq C\left\|\phi_{h}\right\|_{S, K} .
$$

We apply this estimate for $\left\|\psi_{h}\right\|_{K}$ in (4.20) and obtain by means of Young's inequality

$$
A_{h}\left(\phi_{h}, \psi_{h}\right) \geq \sum_{K \in \mathcal{M}_{h}}\left\{\frac{C_{2}}{4}\left\|\phi_{h}\right\|_{S, K}^{2}-C\left\|\phi_{h}\right\|_{K}^{2}\right\} .
$$

Thus, we have shown that there are $h$-independent constants $C_{6}:=C_{2} / 4$ and $C_{7}>0$ such that for all $\phi_{h} \in Y_{h}$ there exists an element $\psi_{h} \in Y_{h}$ satisfying

$$
A_{h}\left(\phi_{h}, \psi_{h}\right) \geq C_{6}\left\|\phi_{h}\right\|_{S}^{2}-C_{7}\left\|\phi_{h}\right\|^{2} .
$$

Now, we are ready to prove our stability result (4.16). Let $\phi_{h}=\left(w_{h}, q_{h}\right)$ be an arbitrary element of $Y_{h}$ and $\psi_{h}:=\left(z_{h}, 0\right) \in Y_{h}$, where $z_{h} \in X_{h}$ is the elementwise defined function from (4.17). Taking the sum of the squares of the $K$-local estimates (4.21) we infer that there exists an $h$-independent constant $C_{8}>0$ such that $\left\|\psi_{h}\right\|_{S} \leq C_{8}\left\|\phi_{h}\right\|_{S}$. Then, we get for the function $\chi_{h}:=C_{7} \phi_{h}+\psi_{h}$ by means of the coercivity (4.15) and (4.22)

$$
A_{h}\left(\phi_{h}, \chi_{h}\right)=C_{7} A_{h}\left(\phi_{h}, \phi_{h}\right)+A_{h}\left(\phi_{h}, \psi_{h}\right) \geq C_{6}\left\|\phi_{h}\right\|_{S}^{2} .
$$


Thus, we obtain from

$$
\left\|\chi_{h}\right\|_{S} \leq C_{7}\left\|\phi_{h}\right\|_{S}+\left\|\psi_{h}\right\|_{S} \leq\left(C_{7}+C_{8}\right)\left\|\phi_{h}\right\|_{S}
$$

for the scaled function $\theta_{h}:=\left(C_{7}+C_{8}\right)^{-1} \chi_{h}$, the estimates $\left\|\theta_{h}\right\|_{S} \leq\left\|\phi_{h}\right\|_{S}$ and

$$
A_{h}\left(\phi_{h}, \theta_{h}\right) \geq C_{6} /\left(C_{7}+C_{8}\right)\left\|\phi_{h}\right\|_{S}^{2}
$$

which concludes the proof of (4.16).

4.3.3. Estimates for the interpolation error. Let $I_{h}^{r}: H^{2}(\Omega)^{d} \cap X \rightarrow X_{h}$ denote the standard Lagrange interpolation operator for the velocity which satisfies the well-known error estimate for all $u \in H^{r+1}(\Omega)^{d} \cap X$ :

$$
\left|u-I_{h}^{r} u\right|_{s, T} \leq C h_{K}^{r+1-s}|u|_{r+1, K} \quad \forall T \in \mathcal{T}_{K}, K \in \mathcal{M}_{h}, s \in\{0,1,2\} .
$$

For $r \geq 2$, we can modify this operator into an operator $\widetilde{I}_{h}^{r}: H^{2}(\Omega)^{d} \cap X \rightarrow X_{h}$ which satisfies the analogous approximation property (4.23) and the additional property for all $u \in H^{2}(\Omega)^{d} \cap X$

$$
\left(\operatorname{div} \widetilde{I}_{h}^{r} u, \bar{q}_{h}\right)_{K}=\left(\operatorname{div} u, \bar{q}_{h}\right)_{K} \quad \forall \bar{q}_{h} \in \mathbb{P}_{0}(K), K \in \mathcal{M}_{h} .
$$

The idea is to add to $I_{h}^{r} u$, for each face $F \subset \partial K$, a face bubble function $I_{h, F}^{r} u$ such that $\left\langle I_{h}^{r} u+I_{h, F}^{r} u, n_{F}\right\rangle_{F}=\left\langle u, n_{F}\right\rangle_{F}$. In the case $d=3$ and $r=2$, we assume that the simplicial submesh $\mathcal{T}_{h}$ generates in the interior of each face $F \subset \partial K$ at least one interior vertex where the face bubble function can be defined as nonzero.

For the pressure, we choose the interpolation operator $\Pi_{h}^{m}: H^{2}(\Omega) \cap M \rightarrow M_{h}$ such that $\left.\left(\Pi_{h}^{m} p\right)\right|_{K}=\Pi_{h}^{m, K}\left(\left.p\right|_{K}\right)$, where $\Pi_{h}^{m, K}: H^{2}(K) \rightarrow U_{m}(K)$ denotes the $L^{2}(K)$ projection onto the composite polynomial space $U_{m}(K)$ defined in $(2.2)$. Note that $\Pi_{h}^{m} p \in M_{h}$ for $p \in M$ due to the $L^{2}$ projection. Since the local mesh $\mathcal{T}_{K}$ of subelements of $K$ is quasi-uniform, we get for $m \geq 1$ and $p \in H^{m+1}(\Omega)$ the error estimate

$$
\left|p-\Pi_{h}^{m} p\right|_{s, T} \leq C h_{K}^{m+1-s}|p|_{m+1, K} \quad \forall T \in \mathcal{T}_{K}, K \in \mathcal{M}_{h}, s \in\{0,1,2\} .
$$

Now, we are ready to prove a local interpolation error estimate in the seminorm $\|\cdot\|_{S, K}$.

Lemma 4.2. Let $r \geq 2$ and $r \geq m \geq 1$ denote the polynomial orders of the finite element spaces $X_{h}$ and $M_{h}$, respectively, and $(u, p) \in X \times M$ be sufficiently smooth such that $u \in H^{r+1}(\Omega)^{d}$ and $p \in H^{m+1}(\Omega)$. Then, it holds for all $K \in \mathcal{M}_{h}$ the estimate

$$
\left\|\left(u-\widetilde{I}_{h}^{r} u, p-\Pi_{h}^{m} p\right)\right\|_{S, K}^{2} \leq C \rho_{K} h_{K}^{2 r}|u|_{r+1, K}^{2}+C \delta_{K} h_{K}^{2 m}|p|_{m+1, K}^{2},
$$

where $\rho_{K}:=\nu+h_{K}+\sigma h_{K}^{2}$.

Proof. Let $K \in \mathcal{M}_{h}$ be an arbitrary composite element and $\eta_{u}:=u-\widetilde{I}_{h}^{r} u$ and $\eta_{p}:=p-\Pi_{h}^{m} p$ denote the single interpolation errors. Then, we have that $\left.\eta_{p}\right|_{K} \in$ $H^{2}(K)+U_{m}(K)$ and (3.12) together with (4.25) yield

$$
J_{K}\left(\eta_{p}, \eta_{p}\right) \leq C \delta_{K}\left\{\left|\eta_{p}\right|_{1, K}^{2}+h_{K}^{2} \sum_{T \in \mathcal{T}_{K}}\left|\eta_{p}\right|_{2, T}^{2}\right\} \leq C \delta_{K} h_{K}^{2 m}|p|_{m+1, K}^{2} .
$$

From $\left.\eta_{p}\right|_{K} \in H^{1}(K)$ and (4.25) we get that $\delta_{K}\left\|\nabla \eta_{p}\right\|_{K}^{2} \leq C \delta_{K} h_{K}^{2 m}|p|_{m+1, K}^{2}$. Using 
the definition (4.11) we obtain

$$
\left\|\left(\eta_{u}, \eta_{p}\right)\right\|_{S, K}^{2} \leq\left(\nu+h_{K}\right)\left|\eta_{u}\right|_{1, K}^{2}+\sigma\left\|\eta_{u}\right\|_{K}^{2}+J_{K}\left(\eta_{p}, \eta_{p}\right)+\delta_{K}\left\|\nabla \eta_{p}\right\|_{K}^{2},
$$

which implies assertion (4.26) by means of (4.25) and (4.23) for $\tilde{I}_{h}^{r}$ instead of $I_{h}^{r}$.

For the subsequent analysis, we need the following norm equivalence.

LEMmA 4.3. For each composite element $K \in \mathcal{M}_{h}$, let

$$
L_{0}^{2}(K):=\left\{q \in L^{2}(K):(q, 1)_{K}=0\right\}
$$

and $U_{m}(K)$ be the composite polynomial space defined in (2.2). Then, for each $m \geq 1$, there exist constants $\underline{c}, \bar{c}>0$ independent of $K \in \mathcal{M}_{h}$ such that

$$
\underline{c} h_{K}\left\|\nabla q_{h}\right\|_{K} \leq\left\|q_{h}\right\|_{K} \leq \bar{c} h_{K}\left\|\nabla q_{h}\right\|_{K} \quad \forall q_{h} \in U_{m}(K) \cap L_{0}^{2}(K) .
$$

Proof. The left part of the inequality (4.28) is a simple conclusion of an inverse inequality. To show the right part we exploit the fact that $\left.q_{h}\right|_{K} \in C^{0}(K) \cap L_{0}^{2}(K)$ such that by means of the integral mean value theorem there exists a point $x^{*} \in \bar{K}$ such that $q_{h}\left(x^{*}\right)=0$. Let $T^{*} \in \mathcal{T}_{K}$ denote a subsimplex such that $x^{*} \in \bar{T}^{*}$ and $c_{K} \in K$ the "center point" such that $c_{K} \in \bar{T}$ for all $T \in \mathcal{T}_{K}$. Applying Taylor's theorem on $\bar{T}^{*}$ we get

$$
\left|q_{h}\left(c_{K}\right)\right|=\left|q_{h}\left(c_{K}\right)-q_{h}\left(x^{*}\right)\right| \leq\left\|\nabla q_{h}\right\|_{0, \infty, \bar{T}^{*}} h_{K} \leq C\left\|\nabla q_{h}\right\|_{T^{*}}\left|T^{*}\right|^{-d / 2} h_{K} .
$$

Now, for each $T \in \mathcal{T}_{K}$ and an arbitrary point $x \in T$, Taylor's theorem, and the fact that the submesh $\mathcal{T}_{K}$ is quasi-uniform imply

$$
\begin{aligned}
\left|q_{h}(x)\right| & \leq\left|q_{h}\left(c_{K}\right)\right|+\left|q_{h}\left(c_{K}\right)-q_{h}(x)\right| \\
& \leq C\left\{\left\|\nabla q_{h}\right\|_{T^{*}}\left|T^{*}\right|^{-d / 2}+\left\|\nabla q_{h}\right\|_{T}|T|^{-d / 2}\right\} h_{K} \leq C\left\|\nabla q_{h}\right\|_{K}|K|^{-d / 2} h_{K},
\end{aligned}
$$

which immediately yields the upper estimate in (4.28).

Finally, we will prove an estimate of the interpolation error in terms of the bilinear form $A_{h}(\cdot, \cdot)$.

Lemma 4.4. Assume the setting of Lemma 4.2 and let $\eta$ denote the interpolation error $\eta=\left(\eta_{u}, \eta_{p}\right)=\left(u-\widetilde{I}_{h}^{r} u, p-\Pi_{h}^{m} p\right) \in X \times M$. Then, it holds for all $\theta_{h} \in Y_{h}$ the estimate

$$
\left|A_{h}\left(\eta, \theta_{h}\right)\right| \leq C\left\{\sum_{K \in \mathcal{M}_{h}}\left(h_{K}^{2 r} \rho_{K}|u|_{r+1, K}^{2}+h_{K}^{2 m} \delta_{K}|p|_{m+1, K}^{2}\right)\right\}^{1 / 2}\left\|\theta_{h}\right\|_{S},
$$

where $\rho_{K}:=\nu+h_{K}+\sigma h_{K}^{2}$.

Proof. Let $\theta_{h}=\left(v_{h}, q_{h}\right) \in Y_{h}$ be an arbitrary element. Then, we have

$$
\begin{aligned}
A_{h}\left(\eta, \theta_{h}\right)=\sum_{K \in \mathcal{M}_{h}}\{ & \nu\left(\nabla \eta_{u}, \nabla v_{h}\right)_{K}+\sigma\left(\eta_{u}, v_{h}\right)_{K}-\left(\operatorname{div} v_{h}, \eta_{p}\right)_{K} \\
& \left.+\left(\operatorname{div} \eta_{u}, q_{h}\right)_{K}+J_{K}\left(\eta_{p}, q_{h}\right)+h_{K}\left(\operatorname{div} \eta_{u}, \operatorname{div} v_{h}\right)_{K}\right\} .
\end{aligned}
$$

The first two terms in the sum can be estimated as

$$
\begin{aligned}
\nu\left|\left(\nabla \eta_{u}, \nabla v_{h}\right)_{K}\right| & \leq \nu\left|\eta_{u}\right|_{1, K}\left|v_{h}\right|_{1, K} \leq\|\eta\|_{S, K}\left\|\theta_{h}\right\|_{S, K}, \\
\sigma\left|\left(\eta_{u}, v_{h}\right)_{K}\right| & \leq \sigma\left\|\eta_{u}\right\|_{K}\left\|v_{h}\right\|_{K} \leq\|\eta\|_{S, K}\left\|\theta_{h}\right\|_{S, K},
\end{aligned}
$$

Copyright $@$ by SIAM. Unauthorized reproduction of this article is prohibited. 
and the third one by

$$
\left|\left(\operatorname{div} v_{h}, \eta_{p}\right)_{K}\right| \leq h_{K}^{-1 / 2}\left\|\eta_{p}\right\|_{K} h_{K}^{1 / 2}\left\|\operatorname{div} v_{h}\right\|_{K} \leq h_{K}^{-1 / 2}\left\|\eta_{p}\right\|_{K}\left\|\theta_{h}\right\|_{S, K} .
$$

To estimate the fourth term we split $\left.q_{h}\right|_{K} \in U_{m}(K)$ as

$$
\left.q_{h}\right|_{K}=\bar{q}_{h}+\tilde{q}_{h} \quad \text { with } \quad \bar{q}_{h} \in \mathbb{P}_{0}(K), \quad \tilde{q}_{h} \in L_{0}^{2}(K) \cap U_{m}(K) .
$$

Using (4.24) and the norm equivalence (4.28) we obtain

$$
\begin{aligned}
\left|\left(\operatorname{div} \eta_{u}, q_{h}\right)_{K}\right| & =\left|\left(\operatorname{div} \eta_{u}, \tilde{q}_{h}\right)_{K}\right| \leq\left\|\operatorname{div} \eta_{u}\right\|_{K}\left\|\tilde{q}_{h}\right\|_{K} \\
& \leq \bar{c}\left\{\nu^{1 / 2}\left|\eta_{u}\right|_{1, K}+h_{K}^{1 / 2}\left\|\operatorname{div} \eta_{u}\right\|_{K}\right\} \min \left(\frac{h_{K}}{\nu^{1 / 2}}, h_{K}^{1 / 2}\right)\left\|\nabla q_{h}\right\|_{K} \\
& \leq C\|\eta\|_{K} \delta_{K}^{1 / 2}\left\|\nabla q_{h}\right\|_{K} \leq C\|\eta\|_{K}\left\|\theta_{h}\right\|_{S, K} .
\end{aligned}
$$

Furthermore, we have

$$
\left|J_{K}\left(\eta_{p}, q_{h}\right)\right| \leq\left\{J_{K}\left(\eta_{p}, \eta_{p}\right)\right\}^{1 / 2}\left\{J_{K}\left(q_{h}, q_{h}\right)\right\}^{1 / 2} \leq\|\eta\|_{S, K}\left\|\theta_{h}\right\|_{S, K}
$$

and

$$
h_{K}\left|\left(\operatorname{div} \eta_{u}, \operatorname{div} v_{h}\right)_{K}\right| \leq\|\eta\|_{K}\left\|\theta_{h}\right\|_{K} .
$$

The assertion (4.29) follows now from the Cauchy-Schwarz inequality, Lemma 4.2, and the interpolation estimate (4.25) for the pressure.

4.3.4. Final error estimate. Now, we are ready to prove our main result on the discretization error of the local CIP discretization.

TheOREM 4.5. Let $\left(u_{h}, p_{h}\right) \in X_{h} \times M_{h}$ denote the solution of the discrete problem (4.9) and $(u, p) \in X \times M$ the solution of the continuous problem (4.3) which is supposed to have the regularity $u \in H^{r+1}(\Omega)^{d}$ with $r \geq 2$ denoting the polynomial order of $X_{h}$ and $p \in H^{m+1}(\Omega)$, where $m$ with $1 \leq m \leq r$ denotes the polynomial order of $M_{h}$. Assume that the mesh $\mathcal{T}_{h}$ is shape regular such that the standard inverse inequalities as well as the condition (2.1) hold. Furthermore, suppose that $\sigma \leq C h^{-1}$. Then, there exists a unique solution $\left(u_{h}, p_{h}\right)$ of the discrete problem (4.9) and there is an $h$-independent constant $C>0$ satisfying the error estimate

$$
\left\|\left(u-u_{h}, p-p_{h}\right)\right\|_{S} \leq C\left(\sum_{K \in \mathcal{M}_{h}}\left\{h_{K}^{2 r} \rho_{K}|u|_{r+1, K}^{2}+h_{K}^{2 m} \delta_{K}|p|_{m+1, K}^{2}\right\}\right)^{1 / 2},
$$

where $\rho_{K}:=\nu+h_{K}+\sigma h_{K}^{2}$ and $\delta_{K}:=\min \left(\nu^{-1} h_{K}^{2}, h_{K}\right)$. Moreover, in the case $\max (\nu, \sigma) \leq C$, it holds for the $L^{2}$-norm error of the pressure the estimate

$$
\left\|p-p_{h}\right\|_{\Omega} \leq C\left(\sum_{K \in \mathcal{M}_{h}}\left\{h_{K}^{2 r} \rho_{K}|u|_{r+1, K}^{2}+h_{K}^{2 m}\left(\delta_{K}+h_{K}^{2}\right)|p|_{m+1, K}^{2}\right\}\right)^{1 / 2}
$$

Proof. Step 1: We show the uniqueness of the discrete solution. Assume that there are two solutions $\left(u_{h}^{i}, p_{h}^{i}\right) \in Y_{h}, i=1,2$, and define $d_{h}=\left(d_{u h}, d_{p h}\right):=$ $\left(u_{h}^{1}-u_{h}^{2}, p_{h}^{1}-p_{h}^{2}\right) \in Y_{h}$. Then, by means of Lemma 4.1 there exists an element $\theta_{h}=\left(\theta_{u h}, \theta_{p h}\right) \in Y_{h}$ such that

$$
\alpha_{1}\left\|d_{h}\right\|_{S}^{2} \leq A_{h}\left(d_{h}, \theta_{h}\right)=0
$$

Copyright (c) by SIAM. Unauthorized reproduction of this article is prohibited. 
This implies

$$
0=\left\|d_{h}\right\|_{S}^{2} \geq\left|d_{u h}\right|_{1, \Omega}^{2}+\sum_{K \in \mathcal{M}_{h}} h_{K}^{2}\left\|\nabla d_{p h}\right\|_{K}^{2}
$$

which leads to the conclusion that $d_{u h}=0$ and $\left.d_{p h}\right|_{K} \in \mathbb{P}_{0}(K)$ for all $K \in \mathcal{M}_{h}$. Since $d_{p h}$ is constant on each macrocell $K$, we infer that $J_{K}\left(d_{p h}, q_{h}\right)=0$ for all $q_{h} \in M_{h}$. Therefore, we get for all $\theta_{h}=\left(\theta_{u h}, \theta_{p h}\right) \in Y_{h}$ that

$$
0=A_{h}\left(d_{h}, \theta_{h}\right)=a\left(0, \theta_{u h}\right)+b\left(\theta_{u h}, d_{p h}\right)-b\left(0, \theta_{p h}\right)=b\left(\theta_{u h}, d_{p h}\right) .
$$

Let $\bar{M}_{h}$ denote the subspace of all piecewise constant pressure functions in $M_{h}$, i.e.,

$$
\bar{M}_{h}:=\left\{q_{h} \in M_{h}:\left.q_{h}\right|_{K} \in \mathbb{P}_{0}(K) \quad \forall K \in \mathcal{M}_{h}\right\} .
$$

Then, it is easy to show that, in the case $r \geq 2$, the pair of finite element spaces $\left(X_{h}, \bar{M}_{h}\right)$ satisfies the $h$-uniform inf-sup condition, i.e., for each function $d_{p h} \in \bar{M}_{h}$ there exists a function $\theta_{u h} \in X_{h} \backslash\{0\}$ such that

$$
b\left(\theta_{u h}, d_{p h}\right) \geq \beta^{*}\left|\theta_{u h}\right|_{1, \Omega}\left\|d_{p h}\right\|_{\Omega},
$$

where $\beta^{*}>0$ is an $h$-independent constant. Thus, from (4.33) it follows $d_{p h}=0$ which concludes the proof of the uniqueness of the discrete solution. Since the discrete problem is equivalent to a finite dimensional linear system of equations, the existence of the discrete solution follows from its uniqueness.

Step 2: To prove the error estimate we split the error as

$$
\left(u-u_{h}, p-p_{h}\right)=\left(u-\widetilde{I}_{h}^{r} u, p-\Pi_{h}^{m} p\right)+\left(\widetilde{I}_{h}^{r} u-u_{h}, \Pi_{h}^{m} p-p_{h}\right)=: \eta+e_{h}
$$

into the interpolation error $\eta=\left(\eta_{u}, \eta_{p}\right) \in X \times M$ and the discrete error $e_{h}=$ $\left(e_{u h}, e_{p h}\right) \in Y_{h}=X_{h} \times M_{h}$. From the regularity assumption we have at least that $p \in H^{2}(\Omega)$. Therefore, the jumps $\llbracket \nabla p \rrbracket_{F}$ vanish for all faces $F$ of the mesh such that $J_{K}\left(p, q_{h}\right)=0$ for all $q_{h} \in M_{h}$ and $K \in \mathcal{M}_{h}$. Together with $\operatorname{div} u=g$ this implies the consistency of our method which leads to the Galerkin orthogonality

$$
A_{h}\left(\left(u-u_{h}, p-p_{h}\right),\left(v_{h}, q_{h}\right)\right)=0 \quad \forall\left(v_{h}, q_{h}\right) \in X_{h} \times M_{h} .
$$

By means of the stability estimate (4.16) applied to $\phi_{h}=e_{h}$ there exists a $\theta_{h} \in Y_{h}$ such that $\left\|\theta_{h}\right\|_{S} \leq\left\|e_{h}\right\|_{S}$ and

$$
\alpha_{1}\left\|e_{h}\right\|_{S}^{2} \leq A_{h}\left(e_{h}, \theta_{h}\right)=A_{h}\left(\left(u-u_{h}, p-p_{h}\right), \theta_{h}\right)-A_{h}\left(\eta, \theta_{h}\right)=-A_{h}\left(\eta, \theta_{h}\right) .
$$

Applying Lemma 4.4, $\left\|\theta_{h}\right\|_{S} \leq\left\|e_{h}\right\|_{S}$, and Young's inequality we infer

$$
\alpha_{1}\left\|e_{h}\right\|_{S}^{2} \leq C \sum_{K \in \mathcal{M}_{h}}\left(h_{K}^{2 r} \rho_{K}|u|_{r+1, K}^{2}+h_{K}^{2 m} \delta_{K}|p|_{m+1, K}^{2}\right)+\frac{\alpha_{1}}{2}\left\|e_{h}\right\|_{S}^{2} .
$$

For the interpolation error $\eta$, we get from Lemma 4.2

$$
\|\eta\|_{S}^{2}=\sum_{K \in \mathcal{M}_{h}}\|\eta\|_{S, K}^{2} \leq C \sum_{K \in \mathcal{M}_{h}}\left(h_{K}^{2 r} \rho_{K}|u|_{r+1, K}^{2}+h_{K}^{2 m} \delta_{K}|p|_{m+1, K}^{2}\right) .
$$

Finally, the estimate (4.31) follows by means of the triangle inequality.

Step 3: It remains to prove the $L^{2}$-norm estimate (4.32) for the pressure. Since the interpolation error $\eta_{p}=p-\Pi_{h}^{m} p$ satisfies the error bound in (4.32), it is sufficient 
to prove this bound for the above defined discrete pressure error $e_{p h}=\Pi_{h}^{m} p-p_{h} \in M_{h}$. In the following, we denote the error bound in $(4.32)$ by $B$, i.e., we set

$$
B:=\left(\sum_{K \in \mathcal{M}_{h}}\left\{h_{K}^{2 r} \rho_{K}|u|_{r+1, K}^{2}+h_{K}^{2 m}\left(\delta_{K}+h_{K}^{2}\right)|p|_{m+1, K}^{2}\right\}\right)^{1 / 2} .
$$

Using the notation of step 2, we split the error into the form

$$
\left(e_{u}, e_{p}\right):=\left(u-u_{h}, p-p_{h}\right)=\left(\eta_{u}, \eta_{p}\right)+\left(e_{u h}, e_{p h}\right) .
$$

In step 2, we have already shown that

$$
\sum_{K \in \mathcal{M}_{h}}\left\{\nu\left|e_{u}\right|_{1, K}^{2}+\sigma\left\|e_{u}\right\|_{K}^{2}+h_{K}\left\|\operatorname{div} e_{u}\right\|_{K}^{2}\right\} \leq\left\|\left(e_{u}, e_{p}\right)\right\|_{S}^{2} \leq C B^{2}
$$

as well as $\sum_{K \in \mathcal{M}_{h}} \delta_{K}\left\|\nabla e_{p h}\right\|_{K}^{2} \leq C B^{2}$ which implies by means of $\max \left(\nu, h_{K}\right) \leq C$ that

$$
\sum_{K \in \mathcal{M}_{h}} h_{K}^{2}\left\|\nabla e_{p h}\right\|_{K}^{2} \leq \sum_{K \in \mathcal{M}_{h}} \max \left(\nu, h_{K}\right) \delta_{K}\left\|\nabla e_{p h}\right\|_{K}^{2} \leq C B^{2} .
$$

We split the error $e_{p h} \in M_{h}$ into the form

$$
e_{p h}=\bar{e}_{p h}+\tilde{e}_{p h} \quad \text { with } \quad \bar{e}_{p h} \in \bar{M}_{h},
$$

such that for all $K \in \mathcal{M}_{h}$ it holds $\left.\tilde{e}_{p h}\right|_{K} \in U_{m}(K) \cap L_{0}^{2}(K)$. Then, we infer from the norm equivalence (4.28) and (4.37)

$$
\left\|\tilde{e}_{p h}\right\|_{\Omega}^{2} \leq \bar{c}^{2} \sum_{K \in \mathcal{M}_{h}} h_{K}^{2}\left\|\nabla \tilde{e}_{p h}\right\|_{K}^{2}=\bar{c}^{2} \sum_{K \in \mathcal{M}_{h}} h_{K}^{2}\left\|\nabla e_{p h}\right\|_{K}^{2} \leq C B^{2} .
$$

Using the Galerkin orthogonality (4.36) for the test function $\theta_{h}=\left(v_{h}, 0\right) \in Y_{h}$, we get

$$
0=A_{h}\left(\left(e_{u}, e_{p}\right),\left(v_{h}, 0\right)\right)=a\left(e_{u}, v_{h}\right)+b\left(v_{h}, e_{p}\right)+\sum_{K \in \mathcal{M}_{h}} h_{K}\left(\operatorname{div} e_{u}, \operatorname{div} v_{h}\right)_{K}
$$

for all $v_{h} \in X_{h}$. Writing $e_{p}=\eta_{p}+\bar{e}_{p h}+\tilde{e}_{p h}$ and using the approximation property $\left\|\eta_{p}\right\|_{\Omega} \leq C B$, the estimate $\max \left(\nu, \sigma, h_{K}\right) \leq C$ as well as the Poincaré-Friedrichs inequality for $v_{h}$ we infer for all $v_{h} \in X_{h}$

$$
\begin{aligned}
b\left(v_{h}, \bar{e}_{p h}\right) & =-a\left(e_{u}, v_{h}\right)-b\left(v_{h}, \tilde{e}_{p h}\right)-b\left(v_{h}, \eta_{p}\right)-\sum_{K \in \mathcal{M}_{h}} h_{K}\left(\operatorname{div} e_{u}, \operatorname{div} v_{h}\right)_{K} \\
& \leq \sum_{K \in \mathcal{M}_{h}}\left\{\nu\left|e_{u}\right|_{1, K}+\sigma\left\|e_{u}\right\|_{K}+\left\|\tilde{e}_{p h}\right\|_{K}+\left\|\eta_{p}\right\|_{K}+h_{K}\left\|\operatorname{div} e_{u}\right\|_{K}\right\}\left|v_{h}\right|_{1, K} \\
& \leq C B\left|v_{h}\right|_{1, \Omega} .
\end{aligned}
$$

Due to the inf-sup condition (4.35) for the spaces $\left(X_{h}, \bar{M}_{h}\right)$ there exists a function $v_{h} \in X_{h} \backslash\{0\}$ such that

$$
\beta^{*}\left\|\bar{e}_{p h}\right\|_{\Omega}\left|v_{h}\right|_{1, \Omega} \leq b\left(v_{h}, \bar{e}_{p h}\right) \leq C B\left|v_{h}\right|_{1, \Omega},
$$

which implies $\left\|\bar{e}_{p h}\right\|_{\Omega} \leq C B$. Finally, by means of the triangle inequality, we obtain the estimate $\left\|e_{p}\right\|_{\Omega} \leq\left\|\eta_{p}\right\|_{\Omega}+\left\|\bar{e}_{p h}\right\|_{\Omega}+\left\|\tilde{e}_{p h}\right\|_{\Omega} \leq C B$. 
TABLE 1

Convergence of the local CIP method with $\gamma_{0}=0.01$ for smooth solution $(\varepsilon=1)$ and polynomial order $r=2$.

\begin{tabular}{c|r||c|c||c|c||c|c}
\hline$\ell$ & $N_{\text {dof }}$ & $e_{L^{2}}^{\text {intp }}$ & Order & $e_{L^{2}}$ & Order & $e_{\text {SD }}$ & Order \\
\hline 1 & 21 & $9.209 \mathrm{e}-04$ & & $7.462 \mathrm{e}-04$ & & $5.381 \mathrm{e}-03$ & \\
2 & 65 & $1.360 \mathrm{e}-04$ & 2.759 & $1.168 \mathrm{e}-04$ & 2.675 & $1.645 \mathrm{e}-03$ & 1.710 \\
3 & 225 & $1.832 \mathrm{e}-05$ & 2.892 & $1.583 \mathrm{e}-05$ & 2.884 & $4.625 \mathrm{e}-04$ & 1.830 \\
4 & 833 & $2.350 \mathrm{e}-06$ & 2.963 & $2.117 \mathrm{e}-06$ & 2.903 & $1.232 \mathrm{e}-04$ & 1.908 \\
5 & 3201 & $2.960 \mathrm{e}-07$ & 2.989 & $2.863 \mathrm{e}-07$ & 2.886 & $3.201 \mathrm{e}-05$ & 1.945 \\
6 & 12545 & $3.713 \mathrm{e}-08$ & 2.995 & $3.916 \mathrm{e}-08$ & 2.870 & $8.211 \mathrm{e}-06$ & 1.963 \\
7 & 49665 & $4.648 \mathrm{e}-09$ & 2.998 & $5.401 \mathrm{e}-09$ & 2.858 & $2.091 \mathrm{e}-06$ & 1.973 \\
8 & 197633 & $5.815 \mathrm{e}-10$ & 2.999 & $7.497 \mathrm{e}-10$ & 2.849 & $5.301 \mathrm{e}-07$ & 1.980 \\
\hline
\end{tabular}

\section{Numerical examples.} $(0,1)^{2}$,

5.1. The transport problem. We consider an example from [6], where $\Omega=$

$$
\beta(x, y):=\frac{1}{\varrho(x, y)}\left(\begin{array}{c}
y+1 \\
-x
\end{array}\right) \quad \text { with } \quad \varrho(x, y):=\sqrt{x^{2}+(y+1)^{2}},
$$

and $\sigma:=0.1$. The exact solution is prescribed as

$$
u(x, y):=\exp \left(-\sigma \varrho(x, y) \arccos \left(\frac{y+1}{\varrho(x, y)}\right)\right) \arctan \left(\frac{\varrho(x, y)-1.5}{\varepsilon}\right)
$$

and the data defined by $f:=\beta \cdot \nabla u+\sigma u=0$ and $g:=\left.u\right|_{\Gamma^{-}}$. Here, the convection field $\beta$ is exactly divergence free and the assumption (3.2) is satisfied with $\mu_{0}=0.1$. The characteristics are the circular lines $\varrho(x, y)=$ const and the inflow boundary part $\Gamma^{-}$consists of the two lines $x=0$ and $y=1$ of the boundary $\partial \Omega$. The coarsest mesh on grid level 1 is derived from a decomposition of $\Omega$ into 2 by 2 squares each of which is subdivided into 4 triangles by drawing the diagonals of the square. Each higher grid level $k+1$ is generated by uniform refinement of the squares from grid level $k$ followed by the triangular subdivision of the new squares. In the following, we present numerical results with the polynomial degree $r=2$ for two situations.

A smooth solution. We choose $\varepsilon=1$ such that the exact solution is very smooth in the sense that the higher order seminorm $|u|_{r+1, \Omega}$ is of moderate size. Here, our aim is to verify the theoretically obtained rates of convergence. Table 1 shows the error norms for the local CIP method with the parameter $\gamma_{0}=0.01$, where

$$
e_{L^{2}}^{\text {intp }}:=\left\|u-I_{h}^{Q_{2}} u\right\|_{L^{2}(\Omega)}, \quad e_{L^{2}}:=\left\|u-u_{h}\right\|_{L^{2}(\Omega)}, \quad e_{\mathrm{SD}}:=\left\|\beta \cdot \nabla\left(u-u_{h}\right)\right\|_{L^{2}(\Omega)}
$$

denote the interpolation error of the Lagrange interpolate $I_{h}^{Q_{2}} u$ in the standard conforming $\mathbb{Q}_{2}$-space over the quadrilateral mesh $\mathcal{M}_{h}$, the discretization error in the $L^{2}$-norm, and the $L^{2}$-error of the streamline derivative, respectively. By $N_{\text {dof }}$ we present for each grid level the number of dofs of the reduced algebraic system after static condensation of the interior dofs in all composite elements. Table 2 presents the analogous results for the standard Galerkin method. For the local CIP method, we see that the $L^{2}$-error is better than the proven order of 2.5 and the error in the streamline deriviative has the optimal order of 2 . However, for the standard Galerkin method, we observe only a suboptimal behavior of the error. 
TABLE 2

Convergence of the Galerkin method $\left(\gamma_{0}=0\right)$ for smooth solution $(\varepsilon=1)$ and polynomial order $r=2$.

\begin{tabular}{c|r||c|c||c|c||c|c}
\hline$\ell$ & $N_{\text {dof }}$ & $e_{L^{2}}^{\text {intp }}$ & Order & $e_{L^{2}}$ & Order & $e_{\text {SD }}$ & Order \\
\hline 1 & 21 & $9.209 \mathrm{e}-04$ & & $7.053 \mathrm{e}-04$ & & $7.073 \mathrm{e}-03$ & \\
2 & 65 & $1.360 \mathrm{e}-04$ & 2.759 & $1.679 \mathrm{e}-04$ & 2.070 & $3.523 \mathrm{e}-03$ & 1.006 \\
3 & 225 & $1.832 \mathrm{e}-05$ & 2.892 & $4.091 \mathrm{e}-05$ & 2.037 & $1.663 \mathrm{e}-03$ & 1.083 \\
4 & 833 & $2.350 \mathrm{e}-06$ & 2.963 & $1.017 \mathrm{e}-05$ & 2.008 & $8.239 \mathrm{e}-04$ & 1.013 \\
5 & 3201 & $2.960 \mathrm{e}-07$ & 2.989 & $2.540 \mathrm{e}-06$ & 2.002 & $4.109 \mathrm{e}-04$ & 1.004 \\
6 & 12545 & $3.713 \mathrm{e}-08$ & 2.995 & $6.348 \mathrm{e}-07$ & 2.001 & $2.053 \mathrm{e}-04$ & 1.001 \\
7 & 49665 & $4.648 \mathrm{e}-09$ & 2.998 & $1.587 \mathrm{e}-07$ & 2.000 & $1.026 \mathrm{e}-04$ & 1.000 \\
8 & 197633 & $5.815 \mathrm{e}-10$ & 2.999 & $3.967 \mathrm{e}-08$ & 2.000 & $5.131 \mathrm{e}-05$ & 1.000 \\
\hline
\end{tabular}

A solution with internal layer. Here, we choose $\varepsilon=10^{-4}$ which implies that the exact solution exhibits an internal layer along the characteristic $\varrho(x, y)=$ 1.5. Moreover, the global higher order seminorm $|u|_{r+1, \Omega}$ is very large such that our theoretical error estimates produce very pessimistic error bounds. In this case, we show first by the "picture norm" in Figure 4 that the stabilized CIP solution $\left(\gamma_{0}=0.01\right)$ exhibits numerical oscillations only near the layer whereas the standard Galerkin solution $\left(\gamma_{0}=0\right)$ suffers from oscillations in a large region. Furthermore, we present in Table 3 the error norms measured on a subdomain $\Omega_{0, h}$ that excludes the internal layer of the exact solution, i.e.,

$$
\Omega_{0, h}:=\left\{x \in \Omega: \exists K \in \mathcal{M}_{h}: x \in \bar{K},\left|\varrho\left(c_{K, 1}, c_{K, 2}\right)-1.5\right| \geq 0.1\right\}
$$

where $c_{K}=\left(c_{K, 1}, c_{K, 2}\right)$ denotes the barycenter of the element $K$.

5.2. The Stokes-Brinkman problem. We consider an example from [5] where $\Omega=(0,1)^{2}$ and the exact solutions for velocity and pressure are prescribed by

$$
u(x, y)=\left(20 x y^{3}, 5 x^{4}-5 y^{4}\right) \quad \text { and } \quad p(x, y)=60 x^{2} y-20 y^{3}-5
$$

such that $g=\operatorname{div} u=0$ and $p \in L_{0}^{2}(\Omega)$. The right-hand side of the Stokes-Brinkman problem (4.1) is defined as

$f(x, y):=-\nu \triangle u(x, y)+\sigma u(x, y)+\nabla p(x, y)=(1-\nu)\left(\begin{array}{c}120 x y \\ 60 x^{2}-60 y^{2}\end{array}\right)+\sigma\left(\begin{array}{c}20 x y^{3} \\ 5 x^{4}-5 y^{4}\end{array}\right)$

where $\nu$ and $\sigma$ are given constants. In Tables 4 and 5 we present the discretization errors for the velocity in the $L^{2}$-norm for the polynomial orders $r=m=2$ and the cases $\sigma=1$ and $\sigma=0$, respectively, on a sequence of uniformly refined grids starting from a $2 \times 2$ quadrilateral macromesh on the unit square $\Omega$. We observe third order of convergence, which is optimal. Furthermore, we see that, for a fixed $h$, the error does not increase when $\nu$ tends to zero. By some simple standard arguments we can rigorously prove that the order of convergence in the $H^{1}$-norm is one order less than in the order in the $L^{2}$-norm. Therefore, we omitted the presentation of the $H^{1}$-norm errors.

In Tables 6 and 7 we present the corresponding discretization errors for the pressure in the $L^{2}$-norm. In the case $\nu=\mathcal{O}(1)$, we observe second order of convergence, which perfectly confirms our theory and which is also well known from the Stokes error analysis where the $L^{2}$-error for the pressure is dictated by the $H^{1}$-error for the velocity. In the case $\nu \lesssim h$, we observe at least the order $\mathcal{O}\left(h^{2.5}\right)$ predicted from our theory and even better in the case of very small $\nu$. Again we omitted the presentation 
solution $\mathrm{u}_{\mathrm{h}}\left(\mathrm{FE}=\mathrm{Qcondens} \mathrm{s}_{2}\right)$, level $=5, \operatorname{dim}\left(\mathrm{V}_{\mathrm{h}}\right)=3201$

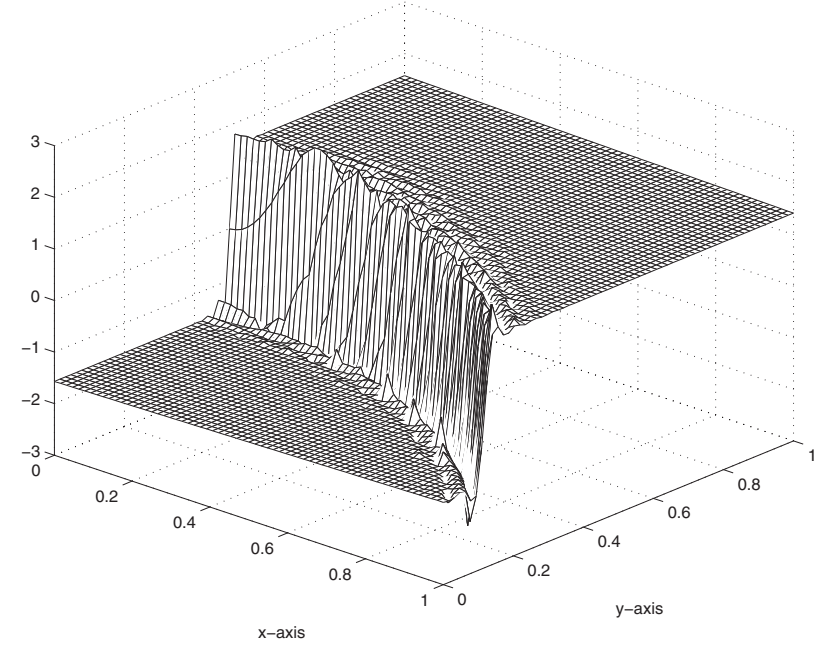

solution $u_{h}\left(F E=Q\right.$ condens $\left.{ }_{2}\right)$, level $=5, \operatorname{dim}\left(V_{h}\right)=3201$

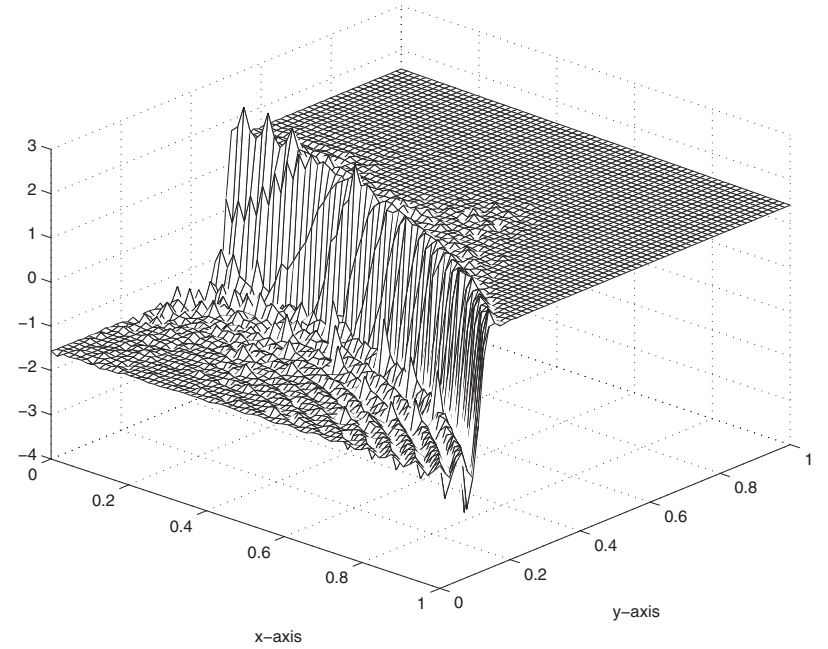

FIG. 4. The case $\varepsilon=10^{-4}, r=2$, and refinement level 5: (upper) CIP solution with $\gamma_{0}=0.01$; (lower) Galerkin solution, i.e., $\gamma_{0}=0$.

TABLE 3

Error norms measured on the subdomain $\Omega_{0, h}$ outside of the layer for the local CIP method with $\gamma_{0}=0.01$ applied to the layer solution $\left(\varepsilon=10^{-4}\right)$ and polynomial order $r=2$.

\begin{tabular}{c|r||c|c||c|c||c|c}
\hline \hline$\ell$ & $N_{\text {dof }}$ & $e_{L^{2}}^{\text {intp }}$ & Order & $e_{L^{2}}$ & Order & $e_{\text {SD }}$ & Order \\
\hline 1 & 21 & $4.436 \mathrm{e}-01$ & & $3.564 \mathrm{e}-01$ & & $3.991 \mathrm{e}-01$ & \\
2 & 65 & $3.048 \mathrm{e}-01$ & 0.541 & $2.269 \mathrm{e}-01$ & 0.651 & $5.509 \mathrm{e}-01$ & -0.465 \\
3 & 225 & $4.412 \mathrm{e}-02$ & 2.788 & $5.159 \mathrm{e}-02$ & 2.137 & $5.376 \mathrm{e}-01$ & 0.035 \\
4 & 833 & $2.117 \mathrm{e}-03$ & 4.381 & $1.897 \mathrm{e}-02$ & 1.443 & $4.303 \mathrm{e}-01$ & 0.321 \\
5 & 3201 & $2.701 \mathrm{e}-07$ & 12.936 & $4.156 \mathrm{e}-03$ & 2.191 & $2.158 \mathrm{e}-01$ & 0.996 \\
6 & 12545 & $2.521 \mathrm{e}-08$ & 3.422 & $5.639 \mathrm{e}-04$ & 2.882 & $6.591 \mathrm{e}-02$ & 1.711 \\
7 & 49665 & $2.848 \mathrm{e}-09$ & 3.146 & $2.558 \mathrm{e}-05$ & 4.462 & $6.542 \mathrm{e}-03$ & 3.333 \\
8 & 197633 & $3.503 \mathrm{e}-10$ & 3.024 & $1.601 \mathrm{e}-07$ & 7.319 & $8.759 \mathrm{e}-05$ & 6.223 \\
\hline
\end{tabular}

Copyright $\odot$ by SIAM. Unauthorized reproduction of this article is prohibited. 
TABLE 4

Discretization error $\left\|u-u_{h}\right\|_{L^{2}(\Omega)}$ and order of convergence for $\sigma=1$ and polynomial order $r=m=2$

\begin{tabular}{l||c|c|c|c|c}
\hline$h$ & $1 / 2$ & $1 / 4$ & $1 / 8$ & $1 / 16$ & $1 / 32$ \\
\hline$\nu=1 e-0$ & $7.577 \mathrm{e}-2$ & $9.461 \mathrm{e}-3$ & $1.186 \mathrm{e}-3$ & $1.485 \mathrm{e}-4$ & $1.857 \mathrm{e}-5$ \\
Order & & 3.002 & 2.996 & 2.998 & 2.999 \\
\hline$\nu=1 e-2$ & $8.007 \mathrm{e}-2$ & $9.828 \mathrm{e}-3$ & $1.214 \mathrm{e}-3$ & $1.504 \mathrm{e}-4$ & $1.868 \mathrm{e}-5$ \\
Order & & 3.026 & 3.017 & 3.013 & 3.009 \\
\hline$\nu=1 e-4$ & $8.043 \mathrm{e}-2$ & $9.806 \mathrm{e}-3$ & $1.216 \mathrm{e}-3$ & $1.515 \mathrm{e}-4$ & $1.891 \mathrm{e}-5$ \\
Order & & 3.036 & 3.011 & 3.005 & 3.003 \\
\hline$\nu=1 e-6$ & $8.044 \mathrm{e}-2$ & $9.806 \mathrm{e}-3$ & $1.216 \mathrm{e}-3$ & $1.512 \mathrm{e}-4$ & $1.885 \mathrm{e}-5$ \\
Order & & 3.036 & 3.012 & 3.007 & 3.004 \\
\hline
\end{tabular}

TABLE 5

Discretization error $\left\|u-u_{h}\right\|_{L^{2}(\Omega)}$ and order of convergence for $\sigma=0$ and polynomial order $r=m=2$.

\begin{tabular}{l||c|c|c|c|c}
\hline$h$ & $1 / 2$ & $1 / 4$ & $1 / 8$ & $1 / 16$ & $1 / 32$ \\
\hline$\nu=1 e-0$ & $7.577 \mathrm{e}-2$ & $9.461 \mathrm{e}-3$ & $1.186 \mathrm{e}-3$ & $1.485 \mathrm{e}-4$ & $1.857 \mathrm{e}-5$ \\
Order & & 3.002 & 2.996 & 2.998 & 2.999 \\
\hline$\nu=1 e-2$ & $8.082 \mathrm{e}-2$ & $9.861 \mathrm{e}-3$ & $1.215 \mathrm{e}-3$ & $1.504 \mathrm{e}-4$ & $1.868 \mathrm{e}-5$ \\
Order & & 3.035 & 3.021 & 3.014 & 3.009 \\
\hline$\nu=1 e-4$ & $8.153 \mathrm{e}-2$ & $9.955 \mathrm{e}-3$ & $1.228 \mathrm{e}-3$ & $1.522 \mathrm{e}-4$ & $1.894 \mathrm{e}-5$ \\
Order & & 3.034 & 3.020 & 3.012 & 3.007 \\
\hline$\nu=1 e-6$ & $8.154 \mathrm{e}-2$ & $9.957 \mathrm{e}-3$ & $1.228 \mathrm{e}-3$ & $1.522 \mathrm{e}-4$ & $1.894 \mathrm{e}-5$ \\
Order & & 3.034 & 3.020 & 3.012 & 3.007 \\
\hline
\end{tabular}

TABLE 6

Discretization error $\left\|p-p_{h}\right\|_{L^{2}(\Omega)}$ and order of convergence for $\sigma=1$ and polynomial order $r=m=2$.

\begin{tabular}{l||c|c|c|c|c}
\hline$h$ & $1 / 2$ & $1 / 4$ & $1 / 8$ & $1 / 16$ & $1 / 32$ \\
\hline$\nu=1 e-0$ & $1.520 \mathrm{e}+0$ & $3.765 \mathrm{e}-1$ & $9.424 \mathrm{e}-2$ & $2.358 \mathrm{e}-2$ & $5.898 \mathrm{e}-3$ \\
Order & & 2.013 & 1.998 & 1.999 & 2.000 \\
\hline$\nu=1 e-2$ & $9.025 \mathrm{e}-2$ & $1.171 \mathrm{e}-2$ & $1.680 \mathrm{e}-3$ & $2.937 \mathrm{e}-4$ & $6.298 \mathrm{e}-5$ \\
Order & & 2.947 & 2.801 & 2.516 & 2.222 \\
\hline$\nu=1 e-4$ & $8.684 \mathrm{e}-2$ & $1.080 \mathrm{e}-2$ & $1.349 \mathrm{e}-3$ & $1.685 \mathrm{e}-4$ & $2.107 \mathrm{e}-5$ \\
Order & & 3.007 & 3.002 & 3.000 & 3.000 \\
\hline$\nu=1 e-6$ & $8.682 \mathrm{e}-2$ & $1.080 \mathrm{e}-2$ & $1.348 \mathrm{e}-3$ & $1.685 \mathrm{e}-4$ & $2.106 \mathrm{e}-5$ \\
Order & & 3.007 & 3.002 & 3.000 & 3.000 \\
\hline
\end{tabular}

TABLE 7

Discretization error $\left\|p-p_{h}\right\|_{L^{2}(\Omega)}$ and order of convergence for $\sigma=0$ and polynomial order $r=m=2$.

\begin{tabular}{l||c|c|c|c|c}
\hline$h$ & $1 / 2$ & $1 / 4$ & $1 / 8$ & $1 / 16$ & $1 / 32$ \\
\hline$\nu=1 e-0$ & $1.512 \mathrm{e}+0$ & $3.760 \mathrm{e}-1$ & $9.421 \mathrm{e}-2$ & $2.358 \mathrm{e}-2$ & $5.898 \mathrm{e}-3$ \\
Order & & 2.007 & 1.997 & 1.998 & 1.999 \\
\hline$\nu=1 e-2$ & $8.797 \mathrm{e}-2$ & $1.151 \mathrm{e}-2$ & $1.660 \mathrm{e}-3$ & $2.920 \mathrm{e}-4$ & $6.286 \mathrm{e}-5$ \\
Order & & 2.935 & 2.793 & 2.507 & 2.216 \\
\hline$\nu=1 e-4$ & $8.626 \mathrm{e}-2$ & $1.078 \mathrm{e}-2$ & $1.348 \mathrm{e}-3$ & $1.685 \mathrm{e}-4$ & $2.107 \mathrm{e}-5$ \\
Order & & 3.000 & 3.000 & 3.000 & 3.000 \\
\hline$\nu=1 e-6$ & $8.626 \mathrm{e}-2$ & $1.078 \mathrm{e}-2$ & $1.348 \mathrm{e}-3$ & $1.685 \mathrm{e}-4$ & $2.106 \mathrm{e}-5$ \\
Order & & 3.000 & 3.000 & 3.000 & 3.000 \\
\hline
\end{tabular}

of the $H^{1}$-norm errors since they are one order less than the $L^{2}$-norm errors. In order to explain the third order of convergence for very small $\nu$ we remark that, in the pure Darcy case $(\nu=0, \sigma=\mathcal{O}(1)$, homogeneous Dirichlet boundary condition for the

Copyright (C) by SIAM. Unauthorized reproduction of this article is prohibited. 
pressure, and no boundary conditions for the velocity), we can rigorously prove by means of a duality argument for the pressure that the $L^{2}$-norm error for the pressure has the order $\mathcal{O}\left(h^{r+1}\right)$ if $m=r \geq 2$.

6. Conclusion and outlook. We have proposed a localized CIP method for stabilization of advection and velocity pressure coupling. The theory was developed in the framework of composite finite elements, but the extension to general subdomains is immediate, with interesting consequences for strongly imposed boundary conditions and $C^{0}$ domain decomposition, in the latter case. For the discretization of the StokesBrinkman equations we exploited the structure of the proposed method to obtain local mass conservation on the macroelement level. The error estimates have been given with explicit dependence on $\nu$, paving the way for a method for the Oseen's equations with local mass conservation on the macroelement level and robustness for high Reynolds numbers.

Another application of the present framework is for convection-diffusion problems with diffusivity $\mu$ that may be discontinuous over the macroelement faces. Observe that in this case the penalty term is not consistent if applied on a face over which the diffusivity jumps. Using this analysis it is straightforward to handle this case in a consistent manner also for the global stabilization operator, if it is modified to take the form

$$
j(u, v):=\gamma_{0} \sum_{F \in \mathcal{F}_{\mathcal{T}_{h}}} h_{F}^{2} \frac{\left|\beta_{F} \cdot n_{F}\right|}{\left.\{\mu\}\right|_{F} ^{2}}\left\langle\llbracket \mu \nabla u \rrbracket_{F}, \llbracket \mu \nabla v \rrbracket_{F}\right\rangle_{F},
$$

where $\mathcal{F}_{\mathcal{T}_{h}}$ denotes the set of interior faces in $\mathcal{T}_{h}, \beta_{F}$ the mean value of $\beta$ over face $F$, and $\left.\{\mu\}\right|_{F}$ the average of the diffusivities on the two sides of the face $F$. The method now remains consistent thanks to the continuity of fluxes.

\section{REFERENCES}

[1] R. Becker And M. BraAck, A finite element pressure gradient stabilization for the Stokes equations based on local projections, Calcolo, 38 (2001), pp. 173-199.

[2] R. BeCKer ANd M. BraAcK, A two-level stabilization scheme for the Navier-Stokes equations, in Numerical Mathematics and Advanced Applications, Springer, Berlin, 2004, pp. 123130.

[3] M. BRAACK AND F. SCHIEWECK, Equal-order finite elements with local projection stabilization for the Darcy-Brinkman equations, Comput. Methods Appl. Mech. Engrg., 200 (2011), pp. 1126-1136.

[4] F. BREZZi And M. Fortin, A minimal stabilisation procedure for mixed finite element methods, Numer. Math., 89 (2001), pp. 457-491.

[5] E. Burman, Pressure projection stabilizations for Galerkin approximations of Stokes' and Darcy's problem, Numer. Methods Partial Differential Equations, 24 (2008), pp. 127-143.

[6] E. BuRman, A posteriori error estimation for interior penalty finite element approximations of the advection-reaction equation, SIAM J. Numer. Anal., 47 (2009), pp. 3584-3607.

[7] E. Burman ANd A. ERn, Continuous interior penalty $h p$-finite element methods for advection and advection-diffusion equations, Math. Comp., 76 (2007), pp. 1119-1140.

[8] E. Burman and P. Hansbo, Edge stabilization for Galerkin approximations of convectiondiffusion-reaction problems, Comput. Methods Appl. Mech. Engrg., 193 (2004), pp. 14371453.

[9] E. Burman And P. Hansbo, Edge stabilization for the generalized Stokes problem: A continuous interior penalty method, Comput. Methods Appl. Mech. Engrg., 195 (2006), pp. 23932410.

[10] T. Chacón Rebollo, M. Gómez Mármol, V. Girault, and I. Sánchez Muñoz, A high order term-by-term stabilization solver for incompressible flow problems, IMA J. Numer. Anal., 33 (2013), pp. 974-1007.

Copyright (c) by SIAM. Unauthorized reproduction of this article is prohibited. 
[11] R. Codina, Stabilization of incompressibility and convection through orthogonal sub-scales in finite element methods, Comput. Methods Appl. Mech. Engrg., 190 (2000), pp. 1579-1599.

[12] A. Ern and J.-L. Guermond, Theory and Practice of Finite Elements, Appl. Math. Sci. 159, Springer-Verlag, New York, 2004.

[13] J.-L. Guermond, Stabilization of Galerkin approximations of transport equations by subgrid modeling, ESAIM Math. Model. Numer. Anal., 33 (1999), pp. 1293-1316.

[14] G. Matthies, P. Skrzypacz, and L. Tobiska, A unified convergence analysis for local projection stabilisations applied to the Oseen problem, ESAIM Math. Model. Numer. Anal., 41 (2007), pp. 713-742.

[15] N. Parolin,, Computational Fluid Dynamics for Naval Engineering Problems, Ph.D. thesis, EPFL, Lausanne, Switzerland, 2004.

[16] H.-G. Roos, M. Stynes, And L. ToBiska, Robust Numerical Methods for Singularly Perturbed Differential Equations, Springer Ser. Comput. Math. 24, 2nd ed., Springer-Verlag, Berlin, 2008.

[17] D. Silvester, Optimal low order finite element methods for incompressible flow, Comput. Methods Appl. Mech. Engrg., 111 (1994), pp. 357-368.

Copyright (C) by SIAM. Unauthorized reproduction of this article is prohibited. 